San Jose State University

SJSU ScholarWorks

Master's Theses

Master's Theses and Graduate Research

Summer 2019

\title{
The Effects of Culture and Gender Roles on the Efficacy of Social Support as a Coping Mechanism for Stress
}

Quy Minh Vu

San Jose State University

Follow this and additional works at: https://scholarworks.sjsu.edu/etd_theses

\section{Recommended Citation}

Vu, Quy Minh, "The Effects of Culture and Gender Roles on the Efficacy of Social Support as a Coping Mechanism for Stress" (2019). Master's Theses. 5049.

DOI: https://doi.org/10.31979/etd.yt7j-7be2

https://scholarworks.sjsu.edu/etd_theses/5049

This Thesis is brought to you for free and open access by the Master's Theses and Graduate Research at SJSU ScholarWorks. It has been accepted for inclusion in Master's Theses by an authorized administrator of SJSU ScholarWorks. For more information, please contact scholarworks@sjsu.edu. 


\title{
THE EFFECTS OF CULTURE AND GENDER ROLES ON THE EFFICACY OF SOCIAL SUPPORT AS A COPING MECHANISM FOR STRESS
}

\author{
A Thesis \\ Presented to \\ The Faculty of the Department of Psychology \\ San José State University \\ In Partial Fulfillment \\ of the Requirements for the Degree \\ Master of Arts
}

by

Quy Vu

August 2019 
(C) 2019

Quy Vu

ALL RIGHTS RESERVED 
The Designated Thesis Committee Approves the Thesis Titled

THE EFFECTS OF CULTURE AND GENDER ROLES ON THE EFFICACY OF SOCIAL SUPPORT AS A COPING MECHANISM FOR STRESS

by

Quy Vu

APPROVED FOR THE DEPARTMENT OF PSYCHOLOGY

SAN JOSÉ STATE UNIVERSITY

August 2019

Cheryl Chancellor-Freeland, Ph.D. Department of Psychology

Megumi Hosoda, Ph.D. Department of Psychology

Arlene Asuncion, Ph.D. Department of Psychology 


\title{
ABSTRACT \\ THE EFFECTS OF CULTURE AND GENDER ROLES ON THE EFFICACY OF SOCIAL SUPPORT AS A COPING MECHANISM FOR STRESS
}

\author{
by Quy Vu
}

Though social support has been thought to be an effective coping mechanism for handling distress, this generalization comes with limitations in that social support is highly intertwined with specific characteristics that could alter both help-seeking behavior and its outcomes. The present study examined the effects of cultural differences on the effectiveness of differing social support sources (i.e., parents versus peers) between European American and Asian/Asian American samples, as well as the influence of gender role orientation on social support outcomes. Ninety-Five European American and Asian/Asian American participants were assigned to either a parent or peer social support letter task prior to a psychosocial stressor. Asian American Values ScaleMultidimensional (AAVS-M) measured cultural values, and the Bem Sex Role Inventory (BSRI) assessed gender orientation. The dependent variable was the change in salivary cortisol levels. Though no significant differences were found for cortisol reactivity between and within cultural groups as a function of social support source, results indicated a potential preference in European Americans, relative to Asians and Asian Americans, for parent support, as seen by lower cortisol reactivity. Additionally, relative to peer support, parent support was marginally more beneficial for European Americans. Lastly, there was no significant interaction between gender roles and social support, including analyses on social support type (i.e., emotional and informational support). 


\section{TABLE OF CONTENTS}

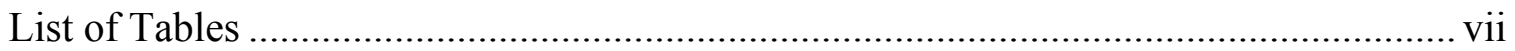

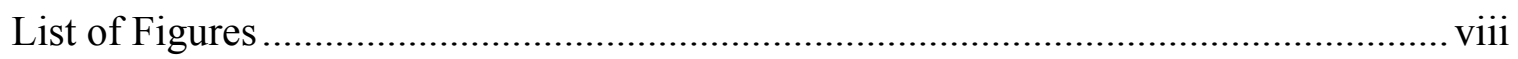

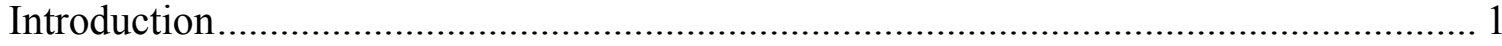

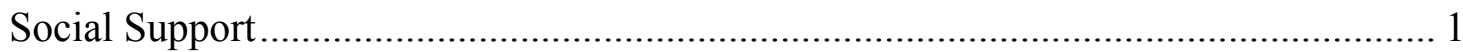

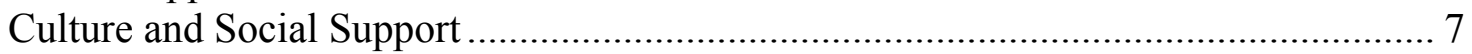

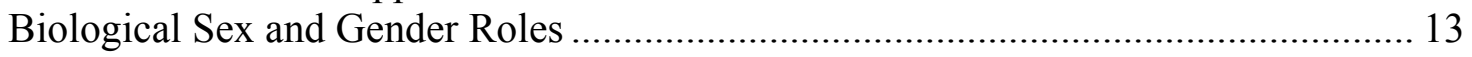

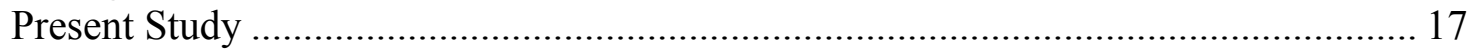

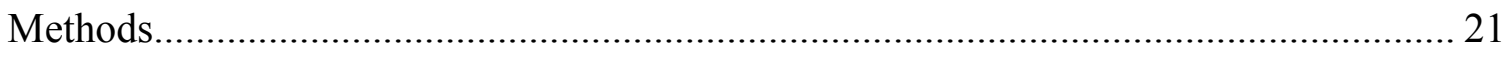

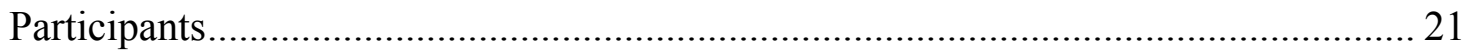

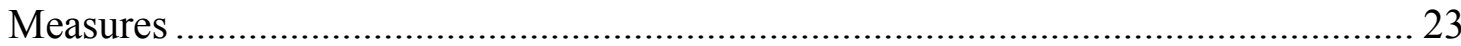

Screening and demographics....................................................................... 23

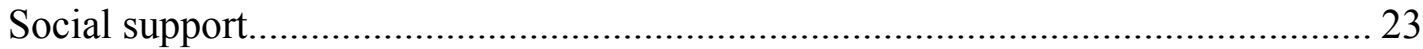

Culture

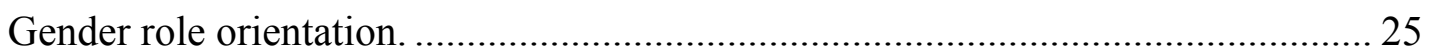

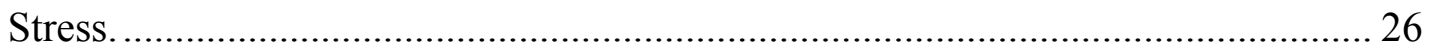

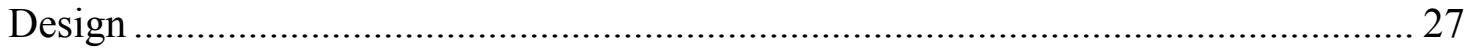

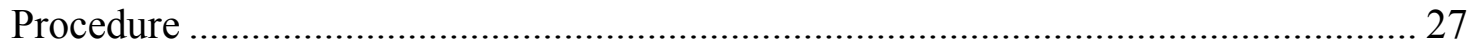

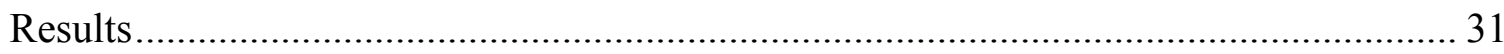

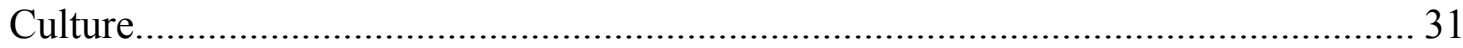

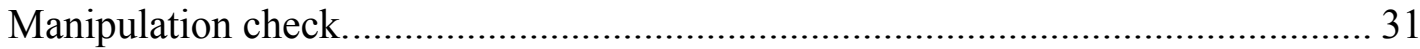

Culture and different social support sources on cortisol reactivity............................. 31

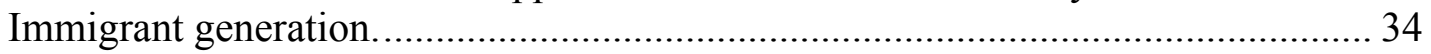

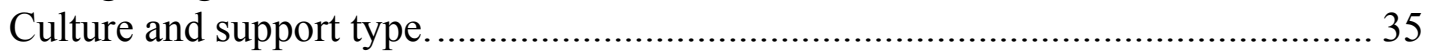

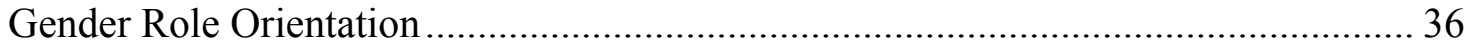

Gender roles and social support sources on cortisol reactivity................................. 36

Gender and social support type......................................................................... 39

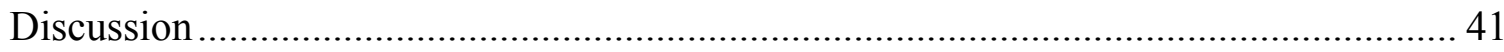

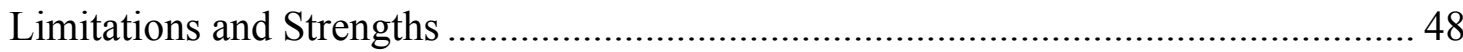

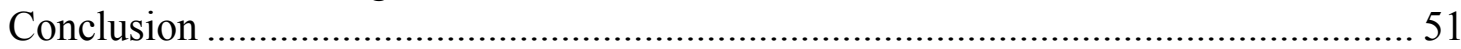

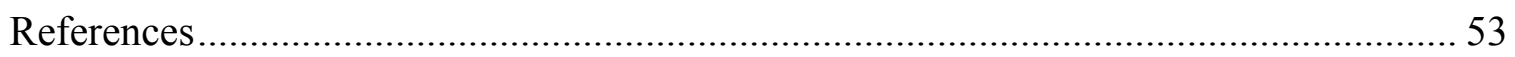

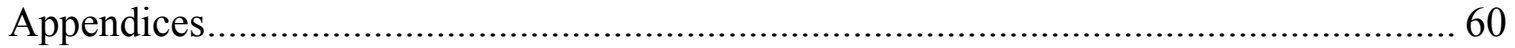

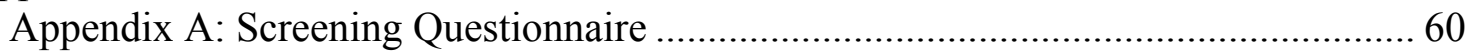

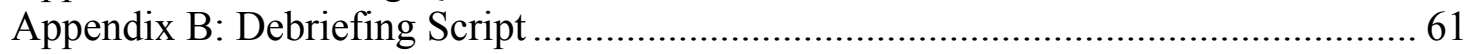

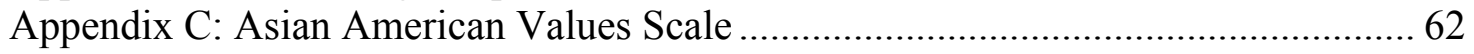

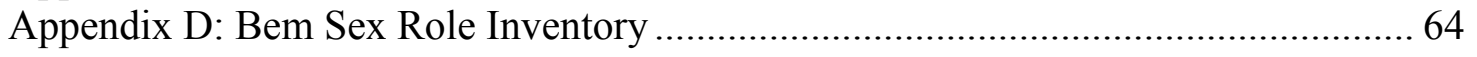




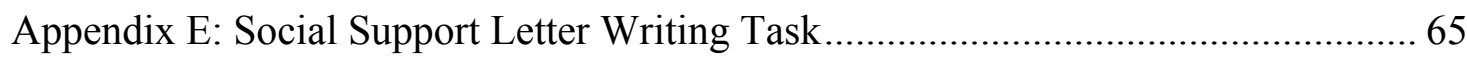




\section{LIST OF TABLES}

Table 1. Culture x Social Support ANCOVA for Cortisol Reactivity..................32

Table 2. Immigrant Generation x Social Support ANCOVA for Cortisol Reactivity.....35

Table 3. Social Support x Gender Roles ANOVA for Cortisol Reactivity...............38 


\section{LIST OF FIGURES}

Figure 1. Breakdown of participant numbers from the experiment and analyses

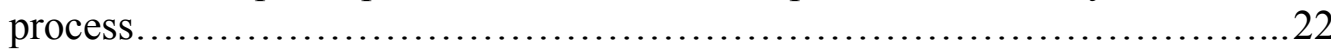

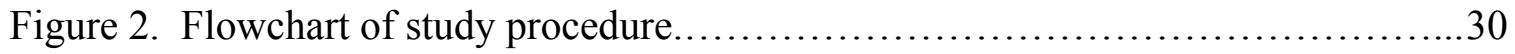

Figure 3. Mean change in cortisol levels for Asian/Asian Americans and European Americans across social support source conditions....................... 33

Figure 4. Preference of support type according to Asians/Asian Americans and European Americans................................................... 36

Figure 5. Frequencies of gender orientations within this sample................... 37

Figure 6. Mean change in cortisol levels across the four gender role orientations.........39 


\section{Introduction}

When encountering a stressor, especially one of a threatening nature, the body's innate response is to initiate the "fight or flight" response. This response involves the activation of the sympathetic nervous system (SNS) and the release of catecholamines, such as epinephrine and norepinephrine, which improves the individual's alertness and vigilance. The activation of the hypothalamic pituitary adrenal (HPA) axis is also initiated, culminating in the release of cortisol (Mason, 1968). Though the SNS is fast and rapid, being an individual's first response to distress, the effects that come with activation of the HPA axis are longer lasting and can be especially detrimental with frequent and sustained activation. More specifically, many detrimental health conditions are related to the effects of prolonged, elevated stress and cortisol levels, including increased anxiety and major depressive disorders, viral and cardiovascular diseases, and immune system dysregulation (Glaser \& Kiecolt-Glaser, 2005; Larzelere \& Jones, 2009). Fortunately, various coping mechanisms to combat distress have been studied for their effectiveness. Most notable among these are exercise, cognitive behavioral therapy, and social support (Berger, 1994; Bryant, Harvey, Dang, Sackville, \& Basten, 1998; Cobb, 1976), the latter of which is reasonably accessible to most. Indeed, a plethora of studies suggests that social support is an effective stress-mitigating factor, but it may depend on several factors, of which culture and gender roles seem particularly important.

\section{Social Support}

As defined by Cobb (1976), social support is the knowledge carried by individuals that they are loved, cared for, and valued by a social network of communication which they 
are mutually a part of. Two notable models have attempted to delineate the processes by which social support influences health: the buffering and main effect models (Cohen \& Wills, 1985). The social support buffering model proposes that social support acts as a protector against the potentially harmful effects of stressful events through two main mechanisms. First, social support may buffer stress by reshaping or preventing negative appraisal of the stressor and by boosting one's ability to cope with the event. In other words, knowing others are willing to provide support may reduce or eliminate the amount of harm or stress that is assessed from a situation. Second, support may provide protection against the physiological reactions of stress through directly influencing the stress response so that the individual is less reactive to the perceived stress. The buffering model is most relevant when support is available while an individual is undergoing stress. In contrast, the main effect model posits a generalized relationship between social support and stress, stating, for example, that an individual with a large social group would likely experience more positive, socially rewarding experiences. As a result, these positive experiences may improve mental health by encouraging healthy self-care practices that could also modify the body's stress response (Cohen \& Wills, 1985).

It should be noted that for social support to properly act as a buffer, the type of support used should match the required resources elicited by the stressful event. To elaborate, there are three distinct types of social support: emotional, informational, and instrumental. Emotional support is the knowledge that a person is accepted and valued for his or her own worth. This type of support may involve the expression of empathy 
toward the individual needing support. Informational support involves help with understanding and defining a problem to cope with the event, and it may otherwise be known as advice or suggestions. Lastly, instrumental support is more tangible and service-related (Cohen \& Wills, 1985).

Not surprisingly, connections between social support and psychological well-being were established early on. Specifically, Aneshensel and Stone (1982) examined the effects of stress and social support on depression. Results revealed that perceived strain and stressful life events were positively correlated with depression. However, close personal relationships and reported perceived support were negatively correlated with depressive symptomology, suggesting that social support may reduce depression. In addition to predicting depression in adult populations, social relationships also influence the risk for depression in other age populations. Emotional support from parents and caretakers during childhood is reported to affect future risk for depression, while, in the elderly, social isolation and reductions in social relationships may be predictors of depression (Kaslow, Deering, \& Racusin, 1994; Oxman, Berkman, Kasl, Freeman, \& Barrett, 1992). Among the college population, students with perceived lower quality social support were at six times the risk for depressive symptoms and were also more likely to experience other mental health issues (Hefner \& Eisenberg, 2009). Social support is also reported to benefit the psychological well-being of individuals experiencing stress and depressive symptoms due to chronic, physical health conditions, such as cardiovascular diseases (Holahan, Moos, Holahan, \& Brennan, 1997; Pennix et 
al., 1998), HIV/AIDS (Turner-Cobb et al., 2002), and rheumatoid arthritis (Goodenow, Reisine, \& Grady, 1990; Taylor, 2007).

The relationship between support and wellness is well established, and studies have consistently reported the health benefits of social relationships and support over the past few decades (e.g., Cohen \& Wills, 1985; Coyne \& Downey, 1991). Early research revealed a relationship between social connections and mortality, with higher mortality rates being linked to individuals with smaller social networks. This link was confirmed when alternate explanations, such as baseline health status, had also been ruled out (Berkman \& Syme, 1979). Further, the absence of social connections as a predictor of mortality demonstrated effect sizes comparable to those of pre-established risk factors, such as smoking and obesity (House, Landis, \& Umberson, 1988).

Subsequent studies continued to show links between improved health and social support, with a particular focus on cardiovascular health. More specifically, research reported solid links between blood pressure and social support, with an association for high social support and lower resting and ambulatory blood pressure scores (Gump et al., 2001; Uchino et al., 1995). The latter is particularly noteworthy because ambulatory blood pressure, beyond resting blood pressure, is a strong predictor of future cardiovascular problems (e.g., Perloff, Sokolow, \& Cohen, 1983). An additional study utilizing a more direct measure of cardiovascular health also found similar links when focusing on disease progression. Imaging techniques used with established coronary artery disease in women revealed faster disease progression with low versus high social support (Wang et al., 2005). 
Another component of health associated with social support is immunity, which bears a direct connection to stress-induced cortisol and its immunosuppressive actions. More specifically, several studies have found links between social support and the lymphocytic immune response. For instance, Lutgendorf and colleagues (2005) examined both psychological distress and social support in women with ovarian cancer, revealing that both variables were associated with changes to the immune response at a cellular level. Specifically, increased natural killer cell activity (i.e., cells critical to the immune defense in cancer patients) was predicted by social support, whereas lower natural killer cell activity was predicted by distress.

Though these studies were significant in uncovering the connection between social support and health conditions, social support had been measured only through means of self-report. Additional research has also examined social support on health outcomes through an experimental lens to more precisely define its impact during stressful situations.

Early studies on the physiological effects of social support in response to stressors utilized measures of cardiovascular reactivity. Lepore, Allen, and Evans (1993) examined the differences in participants' cardiovascular response (i.e., blood pressure) to having no support, the presence of a supportive confederate, or a non-supportive confederate while they delivered a speech. With the sex of the confederate being matched to that of the participants, results revealed that both the participants who delivered their speech alone and those who delivered their speech with a supportive confederate had lower cardiovascular responses to the public speech stressor than did the 
participants who delivered their speech in the presence of a non-supportive confederate. This suggests that being in the presence of a supportive individual is best for reducing stress, while being in the presence of a non-supportive individual is less helpful than being alone for the reduction of stress. A later study by Unchino and Garvey (1996) investigated whether and to what degree the mere knowledge of potential social support could impact cardiovascular reactivity during stress. More specifically, in this study, one group of participants was told that the experimenter would be nearby during their public speech in case they needed help. The outcome was reduced cardiovascular reactivity as compared to the other group of participants who had no access to the experimenter's help.

The described cardiovascular measures are generally interpreted as indices of the sympathetic response to stressors; however, an assessment of the stress hormone, cortisol, is a well-established approach to elucidate the harmful effects of stress. Turner-Cobb and colleagues (2000) examined the association between social support and general cortisol levels in participants with metastatic breast cancer and found that social support predicted lower resting salivary cortisol levels collected over a three-day period, indicating healthier neuroendocrine functioning. Lower basal cortisol levels in women with more social support may suggest that support boosts their coping abilities, which are helpful when handling a cancer diagnosis. Other studies conducted with healthy participants have also found beneficial effects of social support when performing stressful tasks. Findings for cortisol reactivity in response to a laboratory stressor were that participants who delivered a speech in the presence of their friend showed reduced cortisol reactivity 
in comparison to participants who delivered their speech alone (Heinrichs, Baumgartner, Kirschbaum, \& Ehlert, 2003).

\section{Culture and Social Support}

Though research has demonstrated the potential health benefits of social support in both non-stressful and stressful situations, continued research shows various unaccounted factors that affect the outcome of social support. One of the limitations found in these past studies involves the specific cultural context in which most of the studies were conducted. Most studies have been conducted in Western cultures, which proves to be an issue when social support is evaluated from an individualistic perspective. People in individualistic cultures, such as those from the United States, have an independent view on the self, meaning they have a set of defining attributes that influences how they express their personal beliefs and the achievement of personal goals. Relationships are also a notable aspect of individualism because they are chosen by the individual (i.e., more voluntary) and bear few obligations. In contrast, people from collectivistic cultures, such as those from many Asian countries, have an interdependent view on the self, where individuals are connected and bound to others. In these cultures, group goals are prioritized over personal goals, and relationships in collectivistic cultures, in comparison to individualistic relationships, may be less voluntary, entailing a stronger sense of obligation (Adams \& Plaut, 2003; Markus \& Kitayama, 1991). Consistent with this, Asians and Asian Americans are reported less likely to seek social support and more likely to rate the action of seeking social support less positively (Taylor, Sherman, Kim, 
Takagi, \& Dunagan, 2004), spurring further studies to examine cultural differences as a potential moderator for social support effectiveness.

As to why these cultural differences may result in differing social support-seeking behaviors and outcomes, Kim, Sherman, and Taylor (2008) provide three schools of thought. First, Asians and Asian Americans are less likely to directly seek social support partly due to the belief that members of one's close social support group are expected to anticipate the individual's social support needs without explicitly stating so. Second, Asians and Asian Americans may more strongly believe that the responsibility to resolve one's own problems lies within the self. Lastly, Asians and Asian Americans value harmony within the group more strongly than European Americans, and asking for social support from other members of one's group could possibly result in the disruption of the harmony, as well as in negative criticism of the individual asking for help.

The authors posited that this third explanation relating to the maintenance of relationships may be most relevant, again, with Asians and Asian Americans being more cautious when seeking social support as to avoid creating disruptions. Conversely, European Americans are more open to seeking explicit support because their culture encourages the expression of thoughts and needs. Considering these cultural distinctions, Asians and Asian Americans may benefit more from social support that is congruent with their cultural norms, as is further explored in the following research.

As interpersonal consequences are posited to differentially influence collectivists' and individualists' social support-seeking behaviors and outcomes thereof, Kim, Sherman, Ko, and Taylor (2006) experimentally studied the influence of relationships on the 
likelihood of social support seeking between Asians/Asian Americans and European Americans. In this study, the researchers primed relationships by prompting participants to list objectives of the ingroup, outgroup, or the self. Participants in the ingroup condition, for instance, were asked to think and write about a group to which they belonged and then draft a list of the top five goals shared within the group. No differences in social support seeking were found among European Americans across the three primed relationship conditions, as they were less cautious about potential relationship consequences when seeking social support. In contrast, Asians and Asian Americans who were more cautious to avoid negative relational consequences were less likely to seek support if the relationship being primed was related to ingroup goals as compared to if the relationship primed was related to self-goals and outgroup goals. Moreover, the reported effectiveness of social support differed between European Americans and Asians/Asian Americans assigned to the ingroup condition, with the former perceiving social support to be more effective.

Most recently, a study by Tsai, Chiang, and Lau (2016) also revealed culturedependent differences between two coping mechanisms in recovering from stress. Their findings demonstrated the importance of the congruence of general coping mechanisms to the individual's culture. Analyses revealed that Asian Americans were faster at recovering from stress induced by delivering a speech when they were instructed to write about how they could have improved their speech (i.e., self-improvement) as compared to when they were asked to write about how the speech evaluation they received was not credible (i.e., self-enhancement). European Americans, by contrast, more quickly 
recovered from stress when using self-enhancement coping as compared to Asian Americans.

In addition to relationships and coping mechanisms, the form of social support used should also be congruent to the individual's cultural background. While previous research has reported that Asians and Asian Americans are less likely to seek explicit social support (Taylor et al., 2004), there is still the potential for benefiting from other forms of support. Taylor, Welch, Kim, and Sherman (2007) explored the differences in the effectiveness of implicit (taking comfort in knowing one has support available) versus explicit social support (directly using their social support network for advice and help) through the cortisol response of Asians/Asian Americans and European Americans. An analysis revealed an interaction between type of social support and culture, in that Asians and Asian Americans benefited more from implicit social support (i.e., lower cortisol reactivity) than explicit social support, with the opposite result seen for European Americans. Again, underscoring the value of group harmony, Asians and Asian Americans avoid seeking explicit social support in fear of jeopardizing group harmony and personal relationships, two qualities that are prioritized in collectivistic cultures. Conversely, the results within the European American participants were consistent with previous findings that indicate European Americans value verbal expression and disclosure more highly than Asians and Asian Americans (Kim, 2002; Kim \& Sherman, 2007).

In connection to one of the proposed reasons for why cultural differences affect social support seeking and outcomes (Kim et al., 2008), Mojaverian and Kim (2013) studied the 
effectiveness of unsolicited social support, a form of support considered to be more helpful for Asians and Asian Americans because of the cultural belief that social needs should be anticipated by others rather than directly solicited. Though similar to the concept of implicit support studied previously by Taylor and colleagues (2007), the method utilized in this study involved confederates giving advice to participants with or without solicitation. Consistent with the literature, results revealed that Asians and Asian Americans benefited more from unsolicited social support than solicited support. For Asians and Asian Americans, unsolicited support may reinforce feelings of social belonging, whereas soliciting explicit support might risk damaging relational ties. Conversely, European Americans were not affected by the form of social support.

The mutuality of giving and receiving support within relationships may also influence the effectiveness of social support. Wang and Lau (2015) examined differences in cortisol reactivity and perceived stress between Asians/Asian Americans and European Americans after exposure to either mutual (when both individuals in the relationship receive and give support) or non-mutual social support (only one individual receives support). Though there were no differences for the impact of social support condition on the European American participants' stress response (both psychologically and physiologically), Asians and Asian Americans benefited more from support when it was construed as mutual, rather than non-mutual, as demonstrated by both lower cortisol levels and perceived stress. Mutual relationships may be important to Asians and Asian Americans as they are made up of equal contributions to upholding the social bond between the individuals involved. While explicit social support may involve relational 
risks, these potential costs are buffered by reflecting over personal supportive contributions to the relationship. By contrast, there were no differences for the European American participants, as individualism prioritizes the needs of the self, in this case the need for support, over the needs of the group. Interestingly, the researchers also found a trend of Asians and Asian Americans reporting an increased likelihood of going to their peers for social support as compared to their parents, a trend that was reversed in European Americans. This trend for Asians and Asian Americans may in part be related to the mutuality and equality found within peer relationships as compared to the more hierarchical nature of parent and child relationships.

This disparity between peer and parent sources of support was also seen in a study conducted by Wang, Shih, Hu, Louie, and Lau (2010). Not only were Asian Americans, relative to European Americans, less likely to seek social support for both negative and positive life events, when they did solicit support, it was more likely to be from their peers as compared to their parents. This discrepancy between the two social support sources may link back to the obligatory nature of the relationships. While relationships with peers are more voluntary and require fewer obligations, relationships with parents for Asians and Asian Americans are less flexible and can be a more obligatory dynamic, bearing more importance toward keeping the relational ties positive and harmonious. As this study relied on the use of self-report measures, more experimental research including the support source and other potential factors are needed to more carefully define the impact of social support on stress. 


\section{Biological Sex and Gender Roles}

In addition to the effectiveness of social support depending on cultural identification, studies have also revealed differences between the sexes on the likelihood of seeking social support. Indeed, two of the previously discussed studies also found that females were more likely than males to seek social support (Kim et al., 2006; Wang et al., 2010), findings which have been consistently reported over the past few decades (e.g., Tamres, Janicki, \& Helgeson, 2002; Thoits, 1995). Not only are women more likely to seek social support during times of stress, they also more often give and benefit from social support. This trend is seen across developmental stages, with findings that adolescent and collegeaged females were more likely to confide in their same-sex peers for support and actually received more support as compared to their relative male counterparts (Copeland \& Hess, 1995; Ptacek, Smith, \& Zanas, 1992; see Taylor, 2007, for a review). Even though men traditionally report having larger network groups, women tend to be more invested in their relationships, with those relationships holding a greater level of both intimacy and social support (Belle, 1987). Findings for these sex differences in social support behaviors are generally found across differing cultures (Edwards, 1993).

For a more theoretical understanding, the differences in likelihood in seeking support between the two sexes may stem from how they feel about support. Michaud and Warner (1997) found that males reported that they would feel "hurt" or "angry" if they were to receive advice from their friends after sharing their problems. Females, in contrast, reported that they would feel comforted and closer to the friend who provided advice and support. One theory posits that this sex difference occurs as females are more "emotive" 
than males, with studies finding that children as young as two years old demonstrate differences in the amount of emotional expressions displayed during play, with girls displaying more (Malatesta, Culver, Tesman, \& Shepard, 1989). In adulthood, similar findings are seen with the expression of emotions, such that women display a wider variety of facial expressions when expressing emotions in comparison to men (Grossman \& Wood, 1993). This theory suggests that women may find it easier to express their emotions to others during times of distress as compared to men. By contrast, the characteristics of inexpressiveness and self-reliance are reinforced in men from an early age, which makes seeking social support a difficult task, as it clashes with the features of the traditional male-role (Barbee et al., 1993).

Although the finding in two year olds has been interpreted as an innate difference between the sexes regarding emotional expression, parental influence and socialization cannot be completely ruled out. Other bodies of research have revealed that parents behave in ways that elicit more emotions from toddler- and preschool-aged girls than boys. One study by Dunn, Bretherton, and Munn (1987) found that mothers used more words to describe emotional states (e.g., sad, happy) with girls than boys, which may encourage stereotypical stoicism and emotive behavior in boys and girls, respectively. Moreover, the results for sex differences in emotional expressions found in the Grossman and Wood (1993) study were larger among individuals with stronger gender-related stereotypical emotional behavior. Specifically, women who more strongly believed in the stereotype that the average woman feels emotions more intensely than men also reported experiencing emotions more intensely themselves. For men, those who also 
more strongly believed in that stereotype reported less intensity of their emotions, which corresponds with the male stereotype of stoicism. These gender stereotypes are also seen in the judgment from other individuals, such that women disclosing emotional information about themselves were rated more positively, but the same disclosing behavior in men negatively impacted their ratings (Collins \& Miller, 1994). In sum, these studies demonstrate the reinforcement of gender-specific stereotyped behaviors in the realm of social support seeking and receiving, suggesting these sex differences may not only be innate.

One way to more precisely dissect the role of socialized gender roles on supportseeking behaviors and outcomes is to disregard biological sex. Though gender roles are the attitudes and behaviors that are acceptable for individuals based on their (perceived) sex, it is possible for people to take on gender roles that are not traditionally congruent with their sex (Bem, 1974a; Kagan, 1964). Instead, splitting the sexes into individuals who are more feminine or masculine has revealed differences in an individual's likelihood to seek social support, regardless of biological sex. For instance, one study (Reevy \& Maslach, 2001) found that femininity (in both sexes) was associated with seeking and receiving social support, especially emotional support, as well as seeking and receiving support from women. Masculinity, in contrast, was associated only with receiving instrumental support. The researchers concluded that the traits involved with masculinity (e.g., independence, stoicism) may not equip these people for seeking and receiving social support, especially emotional support. They proposed that socialization of masculine individuals prepares them more for practical approaches to problem solving. 
As a result, these individuals are likely to prefer tackling issues head on, which may explain the association between masculinity and receiving a more tangible form of support (Reevy \& Maslach, 2001). A subsequent study examining coping strategies used among late adolescents also found comparable results, such that problem-focused and emotion-focused coping strategies were endorsed more by highly masculine and feminine individuals, respectively (Renk \& Creasey, 2003).

While the previous studies depicted the socialization of femininity and masculinity to influence social support-related behaviors, there is a dearth of research on individuals who neither score as feminine nor masculine. Research by Bem (1974a) and Spence, Helmreich, and Stapp (1975) suggests that masculinity and femininity act as two independent scales. Depending on how individuals score on each scale, they are categorized into one of four gender role orientations, which include categories for androgyny (high scores for masculinity and femininity scales) and undifferentiation (low scores for both scales) in addition to femininity and masculinity. Androgynous-oriented individuals, therefore, have high amounts of both masculine and feminine attributes, whereas undifferentiated-oriented individuals have fewer attributes of both. Spence and colleagues (1975) and Bem (1977) posit that the category of androgyny is connected to better psychological health, with androgynous individuals scoring highest on self-esteem measures, and subsequent studies support this suggestion (e.g., Glazer \& Dusek, 1985; O’Connor, Mann, \& Bardwick, 1978). Surprisingly, undifferentiated individuals scored lowest on self-esteem and were also observed to be the least responsive in studies where participants interacted with kittens and five-month old babies (Bem, Martyna, \& Watson, 
1976). Though no studies have examined the interaction between all four gender orientations and social support, a recent study revealed that undifferentiated individuals were more likely to utilize an unhealthy coping technique. Specifically, they were more likely than those with the other gender role orientations to use alcohol as a coping mechanism (Fugitt, Ham, \& Bridges, 2017). Despite these coping differences between the gender orientations, there remains an absence of literature exploring the impact of social support for persons who are gender identified beyond traditional labels of femininity and masculinity.

\section{Present Study}

Though previous studies have revealed both a difference in social support source preference (i.e., parents versus peers) between cultures, and a discrepancy of social support-seeking and receiving behavior between feminine and masculine individuals, very few studies, if any at all, have looked beyond self-report measures in assessing stress with these specific variables. Further, no studies have considered non-traditional gender orientations in relation to stress and the benefits of social support. Being that chronic stress and prolonged HPA activation are connected to ill health outcomes, exploring the possible moderating effects of culture and gender role identification on support is vital to improving our understanding of treatment for individuals with various health conditions.

In the current study, I attempted to bridge these gaps in the literature for social support source and culture, as well as for gender orientation and social support. To elaborate, I assessed the effectiveness of two different sources of social support (i.e., parent and peer) for individuals from different cultural backgrounds (i.e., individualistic and collectivistic 
cultures) prior to exposure to a psychosocial stressor (Trier Social Stress Test; TSST). Social support source was manipulated by instructing participants to write a letter to either a parent or a peer seeking advice for the TSST. With the same psychosocial stress paradigm, I also assessed the relationship of four gender orientations (i.e., feminine, masculine, androgynous, and undifferentiated), regardless of support source and biological sex, on the efficacy of explicit social support in attenuating stress. Lastly, I examined the relationship between social support type (i.e., emotional and informational) and gender orientation and culture, with the culture and support type interaction being more exploratory due to a lack of previous research on the topic. Support type was not manipulated, but rather, coded from a writing task. The following are the hypotheses that are tested in the present study.

Hypothesis 1. Asians and Asian Americans will benefit more from asking their peers for advice as compared to asking their parents. In other words, Asians and Asian Americans will show relatively lower levels of salivary cortisol and stress in the peer condition.

Hypothesis 2. European Americans will benefit more from asking their parents for advice as compared to their peers. Both Hypotheses 1 and 2 are based on the previous finding that Asians and Asian Americans are more likely to seek social support from their peers than from their parents, while European Americans are more likely to seek social support from their parents than from their peers (Wang et al., 2015; Wang et al., 2010). To reiterate, in collectivism, an individual's relationship with his or her parents is often more obligatory and involuntary than that with his or her peers, which may 
dissuade the individual from seeking parental social support as to maintain relational ties and harmony. Additionally, previous research also revealed differences in cortisol reactivity between Asians/Asian Americans and European Americans as a function of social support form (e.g., explicit versus implicit support), results of which have shown that individuals benefited more from social support when the method was congruent with their culture (e.g., Taylor et al., 2007; Tsai et al., 2016; Wang et al., 2015).

Hypothesis 3. In turn, because the general mode of social support seeking in this study is explicit in nature, Asians and Asian Americans will benefit less overall from the social support task (regardless of source), as demonstrated by higher cortisol concentrations relative to European Americans.

Hypothesis 4. However, receiving social support will be, overall, more beneficial than receiving no support at all. This will be demonstrated as higher cortisol reactivity in the control group for participants from both cultures relative to those in the social support groups.

Hypothesis 5. Feminine individuals will benefit more from social support and will, therefore, demonstrate lower stress responses in comparison to masculine individuals as well as to individuals in the no social support group. This is because feminine individuals are more likely to seek social support than masculine individuals (Reevy et al., 2001). Regarding the remaining two gender roles, as the literature lacks research on the interaction between androgynous and undifferentiated orientations with social support, it was difficult to form a concrete hypothesis. But as a previous study 
revealed that undifferentiated-oriented individuals were more likely to use unhealthy coping behaviors (Fugitt et al., 2017), I hypothesize that these individuals will benefit less from social support than feminine individuals and will, therefore, have higher cortisol reactivity.

Hypothesis 6. Androgynous individuals will display a lower stress response following the stressor as compared to other individuals, as Bem (1974a) has suggested androgynous-oriented individuals to be most psychologically healthy. However, the lack of research on the relationship between androgyny and social support makes it difficult to hypothesize how these individuals will respond to social support in comparison to the other gender roles.

Hypothesis 7. Lastly, as this study does not directly prompt participants towards using any specific support type, data for both the usage of emotional and/or informational support will also be available. Here, I hypothesize that social support type (i.e., emotional, informational, and both) will influence how beneficial social support is in mitigating stress for the different gender identities. Specifically, feminine and masculine individuals will benefit most from seeking emotional and informational support, respectively, as similarly demonstrated in past research (Reevy \& Maslach, 2001; Renk \& Creasey, 2003). As there are few to no studies assessing non-binary gender roles within the field of social support, it was difficult to form hypotheses for support type use among undifferentiated and androgynous individuals. Analyses on support type and the two non-binary gender roles, as well as culture, will be more exploratory in nature. 


\section{Methods}

\section{Participants}

One hundred and forty-four Asian/Asian American and European American participants ranging from the ages of 18 years to 27 years were recruited from the Psychology 1 research pool at San José State University for course credit. Of the 144 participants, 95 completed all of the study criteria and were included in the final analyses. This final sample consisted of 45 Asians and Asian Americans (26 female and 19 male) and 50 European Americans (33 female and 17 male). Of the Asian and Asian American participants who responded to the demographic question regarding ethnic region $(n=21)$, 33\% were East Asian and 67\% were Southeast Asian. Regarding immigrant generations, 33\% of Asian and Asian American participants reported being a first generation American (i.e., foreign-born U.S. resident), 59\% reported being second generation American (i.e., U.S.-born to foreign-born parents), and 8\% reported being third generation American (i.e., U.S.-born to foreign-born grandparents). Among European American participants, $8 \%$ were first generation, $14 \%$ were second generation, $64 \%$ were third generation American and beyond, and 14\% opted not to respond. All participants underwent the TSST. Participants were excluded from participating if they reported having any history of mood or anxiety and inflammatory or endocrine disorders. At-risk populations, such as pregnant women and military veterans, were also exempt, the latter due to possible underlying Post-Traumatic Stress Disorder (PTSD). Participants were required to abstain from exercise and eating or drinking anything besides water for an hour prior to the experiment, as those activities may affect salivary cortisol samples. 
Informed consent was obtained in writing, following screening for the previously mentioned exclusion criteria. Refer to Figure 1 for a summary of the numbers of excluded and included participants throughout the experiment and analysis process, as well as the sample sizes of the randomly assigned social support conditions.

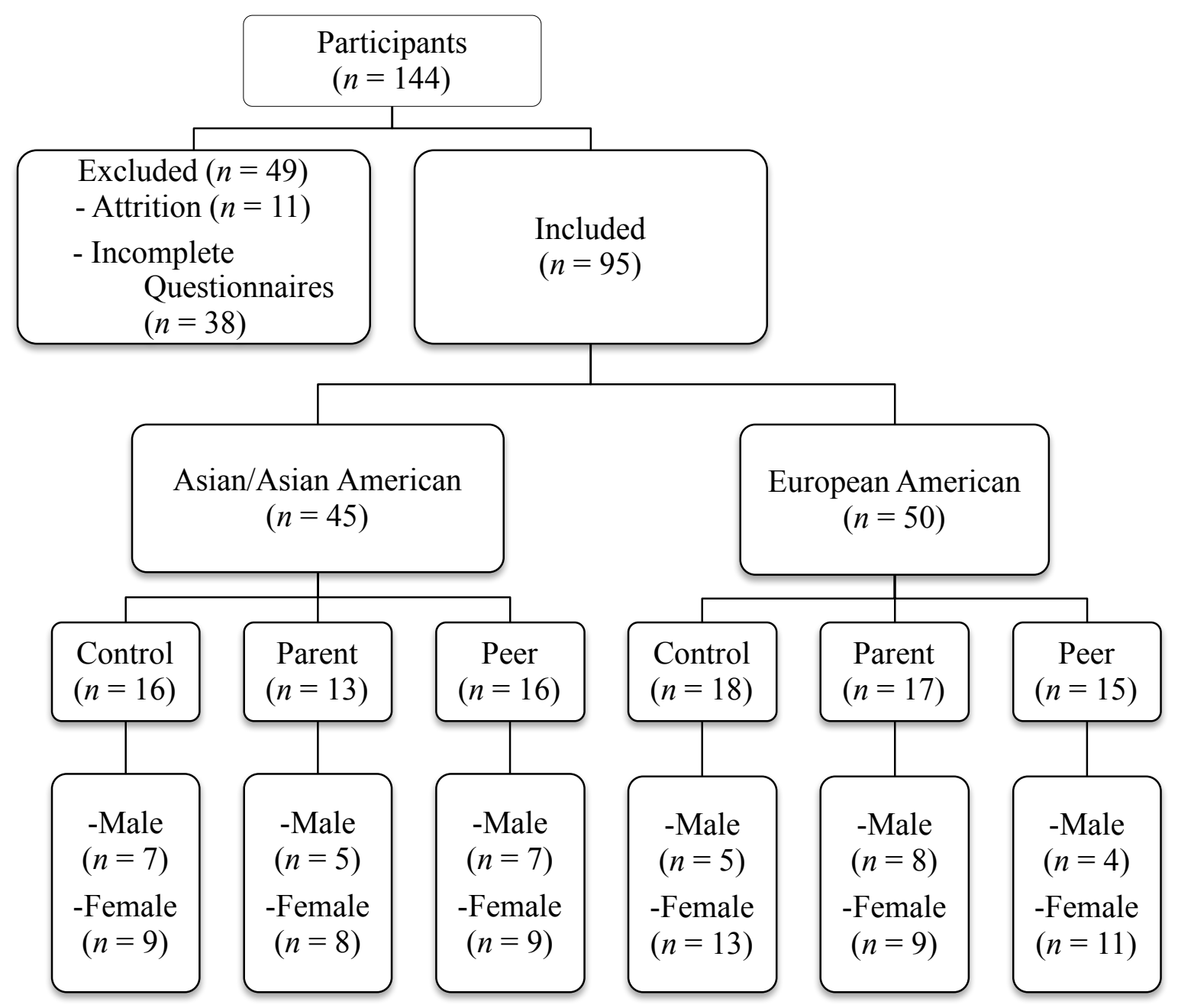

Figure 1. Breakdown of participant numbers from the experiment and analyses process. 


\section{Measures}

Screening and demographics. A screening questionnaire was used to ensure participants met the study requirements (see above for criteria or Appendix A). A demographics questionnaire was included to collect information on age, generation, ethnicity, and biological sex.

Social support. Social support was manipulated with a revised version of methods used in the Taylor et al. (2007) and Wang et al. (2015) studies. Participants were randomly assigned to either the no support, parent support, or peer support group. Those in the parent support group were asked to think about one of their parents and to write a letter to that parent asking for advice on the TSST. Participants were also asked to think about the advice their parent would respond with. Those in the peer support group received similar instructions, with the only difference being that they had to write a letter to one of their peers rather than to their parent. Lastly, those in the no support group were asked to think and write about concerns they had regarding the TSST. Though the letter writing manipulation was borrowed from the earlier studies, the control condition was adapted to fit the current research interests and to address past criticisms. Originally, Taylor and colleagues (2007) used a similar writing task to evoke explicit social support, but a different writing prompt for the control group (i.e., describing a campus tour) was reported to have acted as a distractor instead of a control, and thus diminished anticipatory stress. The control group's focus on the imminent psychosocial stressor (i.e., the TSST) intended to control for this. 
In sum, the writing manipulation was designed to focus attention on the TSST and to evoke a sense of explicit social support; however, the task left it open as to whether the support was emotional and/or informational in nature. To determine the specific type of support, definitions of emotional and informational support were used to code participants' letters. To elaborate, letters that included venting of emotions or seeking encouragement (e.g., "I'm nervous," or "Support me during this stressful event") were coded as emotional. Letters that asked for practical tips on job interviews or public speaking (e.g., "What qualifications should I mention?") were coded as informational. Letters that included both types were coded as both. Participants were given three minutes to write the letter. It should be noted that each letter was examined to ensure completion and adherence to the prompt.

Culture. Adherence to cultural values as well as differences between cultural values was measured by the Asian American Values Scale-Multidimensional (AAVS-M; Kim, Li, \& Ng, 2005). The AAVS-M consisted of five subscales (i.e., collectivism, conformity to norms, emotional self-control, family recognition through achievement, and humility), with 42 total items. The collectivism subscale consisted of seven items (e.g., "The welfare of the group should be put before that of the individual"). The conformity to norms subscale consisted of seven items (e.g., "One should recognize and adhere to the social expectations, norms, and practices"). The emotional self-control subscale consisted of eight items (e.g., "It is better to hold one's emotions inside than to burden others by expressing them"). The family recognition through achievement subscale consisted of 14 items (e.g., "One should achieve academically since it reflects on one's 
family"). And lastly, the humility subscale consisted of six items (e.g., "One should not sing one's own praises"). The internal reliability of each subscale from the original study were $.92, .86, .84, .86, .91$, and .80 , respectively. A reliability analysis was also conducted for the current study, and Cronbach's alpha revealed good internal consistency for the overall scale, $\alpha=.79$. Participants were asked to rate the extent to which they agreed with each statement by using a 7-point Likert scale $(1=$ strongly disagree, $7=$ strongly agree). The scale was created to measure values enculturation, specifically the differences in values between Asian Americans and European Americans.

Gender role orientation. Gender role orientation was measured by the Bem Sex Role Inventory (BSRI; Bem, 1974b). The BSRI is a 60-item inventory with items relating to both the feminine and masculine stereotypes. For example, items that fall under the masculine sex role are aggressive and dominant, while items like gentle and affectionate fall under the feminine sex role. The BSRI also includes neutral items, such as adaptable and truthful. Participants were asked to rate the extent to which each of the items described themselves $(1=$ never or almost never true, $7=$ almost always true $)$. The masculine and feminine items make up two independent scores. Scoring high on femininity and low on masculinity classified individuals as feminine, and scoring low on femininity and high on masculinity classified individuals as masculine. Androgynous and undifferentiated individuals scored high-high and low-low, respectively. Psychometric analyses have revealed the high internal consistency (masculinity $\alpha=.86$ and femininity $\alpha=.82$ ) and test-retest reliability of the BSRI (masculinity and femininity $r=.90$ and androgyny $r=.93$ ). Using Cronbach's alpha, reliability analyses for data 
from the current study revealed good internal consistency for the items in both the masculine $(\alpha=.87)$ and feminine $(\alpha=.82)$ scales.

Stress. Stress was induced in participants through the Trier Social Stress Test (TSST). This stressor spanned a total of 15 minutes, with time given for instructions, preparation, and the task itself. Participants were first instructed of the speech portion of the task, which was to deliver a speech on their ideal job. Participants were then given three minutes to prepare for their speech with the option to write down notes about what they wanted to say. However, participants were not allowed to bring the notes with them to deliver their speech. The TSST itself consisted of a five-minute speech on their ideal job and a mental arithmetic task that entailed counting aloud backwards from a large number to 0 , subtracting by a specified amount. The task was to be completed by participants while they were standing in front of a panel of judges who were trained to maintain a neutral tone and expression throughout the task, offering nothing more than scripted answers if prompted by the participants (Kirschbaum, Pirke, \& Hellhammer, 1993). Previous research revealed the efficacy of the TSST as an acute stressor, showing a two to three time increase in cortisol levels among 70 to $80 \%$ of participants who underwent the stressor (Kudielka, Hellhammer, Kirschbaum, Harmon-Jones, \& Winkielman, 2007).

Stress was measured through two salivary cortisol samples collected from participants, one prior to the TSST (baseline) and the second five minutes following the TSST (peak). Using the Salivette ${ }^{\circledR}$ Cortisol devices (Sarstedt AG \& Co., Nümbrecht, Germany), participants chewed on a cotton cylinder for one minute prior to spitting the cotton back into the tube. Kirschbaum and Hellhammer (1994) previously demonstrated the 
reliability and validity of measuring cortisol through saliva samples. Saliva was extracted from the cylinder tubes through centrifuging prior to analyses for cortisol concentrations with Salimetrics Cortisol ELISA kits (Salimetrics, LLC, State College, PA). Stress reactivity ( $\Delta$ Cortisol) was calculated by subtracting baseline cortisol concentration from post-stressor cortisol concentration and is presented in $\mu \mathrm{g} / \mathrm{dL}$ (micrograms per deciliter).

\section{Design}

For this study, a 4 × $3 \times 2$ factorial design with one dependent variable was used. Two of the three factors were quasi-independent variables, those two specifically being culture (i.e., Asian/Asian American and European American) and gender role identification (i.e., feminine, masculine, androgynous, and undifferentiated). The remaining independent variable was the social support condition, with the three levels being no social support, parent support, and peer support. All independent variables were between-subjects, and all analyses were conducted using IBM SPSS Statistics version 23. Though the design consisted of three factors, the analyses did not include a three-way analysis of variance (ANOVA) due to the attrition and omission of participants from compliance issues, resulting in a smaller sample size than anticipated. Lastly, the dependent variable $\Delta$ Cortisol $(\mu \mathrm{g} / \mathrm{dL})$ was determined by subtracting participants' baseline cortisol levels from their peak cortisol levels and was used to measure change in cortisol.

\section{Procedure}

On the day of the experiment, participants arrived at the research suite some time during 12:00 p.m. to 6:00 p.m. (to control for cortisol's circadian rhythm; Kudielka, 
Schommer, Hellhammer, \& Kirschbaum, 2004) and went through a quick screening checklist. This checklist was used to ensure they had abided by the agreement to abstain from exercise and eating and drinking anything besides water for an hour prior to their arrival. This checklist also ensured that the participants were healthy and had no history of mood or anxiety and inflammatory or endocrine disorders. Once they passed the screening, they gave written informed consent before providing the experimenter a baseline saliva sample. Participants were asked to chew on a piece of cotton from a Salivette tube for one minute and to spit it back into the tube afterward. Next, participants were led into an interview room to receive instructions for the TSST. Within the interview room, the participants were introduced to two judges in white lab coats, both of whom were trained to maintain both a neutral facial expression and neutral tone when giving scripted instructions during the actual task, as to not provide participants with any type of feedback. The experimenter briefly summarized the speech task of the TSST, telling participants that they were expected to present a speech on their ideal job and why they were the best candidate for the job. The experimenter also pointed the participants' attention toward a video camera set up to the side, telling the participants that their speech would be recorded but deleted upon review. Following these initial instructions, participants were led back to the preparation room, in which they were given three minutes to prepare their speech with the option to jot down notes.

After the participants completed their preparations, the experimenter returned to instruct them on the social support letter writing exercise, which took an additional three minutes to complete. Participants were randomly assigned to one of three social support 
conditions (i.e., parent, peer, or no social support) by drawing slips from an envelope prior to the social support letter task. This method of condition assignment was, in addition to ensuring randomization, to reduce any risk of experimenter bias. Those in the social support groups were instructed to write a letter asking for advice for the TSST they would be undergoing. The recipient of the letter was either one of their parents or one of their peers, depending on which of the two social support groups they were assigned to. Participants in the control group were instructed to think and write about concerns they had for the upcoming TSST. Participants were also informed that though the letters would not be thoroughly analyzed, they would be checked to ensure the participants adhered to the prompt. This was done as an incentive for the participants to maintain on task (see Appendix E for task prompts).

Once the letter writing exercise ended, the experimenter led the participants back to the interview room and left the participants alone with the two judges. The main judge then repeated the instructions for the TSST. During the five-minute speech, each period of 20 seconds of silence from the participants resulted in the main judge prompting the participants to continue speaking. During this time, the assistant judge recorded handwritten notes about the participants' speech, which was meant to add onto the participants' distress. After the speech, the main judge gave further instructions for a mental arithmetic task in which the participants were asked to count aloud backwards from 2083 to 0 , subtracting by 13 each time. Every time the participants made a mistake, the main judge prompted them to start again from the beginning. In addition to the 
implication of there being no time limit for the arithmetic task, as the participants were asked to count to 0 , participants were also not told of this task beforehand.

With stress onset being the introduction to the TSST, approximately 17 minutes had elapsed by the time the participants finished the TSST. Back in the preparation room, the AAVS-M was administered, and participants were given five minutes to complete the items. Following the five minutes, the experimenter returned to the room to collect the second saliva sample to capture peak levels of stress. Immediately after the saliva sample, the experimenter administered the BSRI and a demographics survey, which took another 10 minutes to complete. The participants were then debriefed about the intentions of the study and provided with information for counseling centers on campus for precautionary measures due to the nature of the TSST. See Appendix B for the debriefing script. For a summary of the experimental process, the flowchart in Figure 2 depicts a condensed version of the procedure.

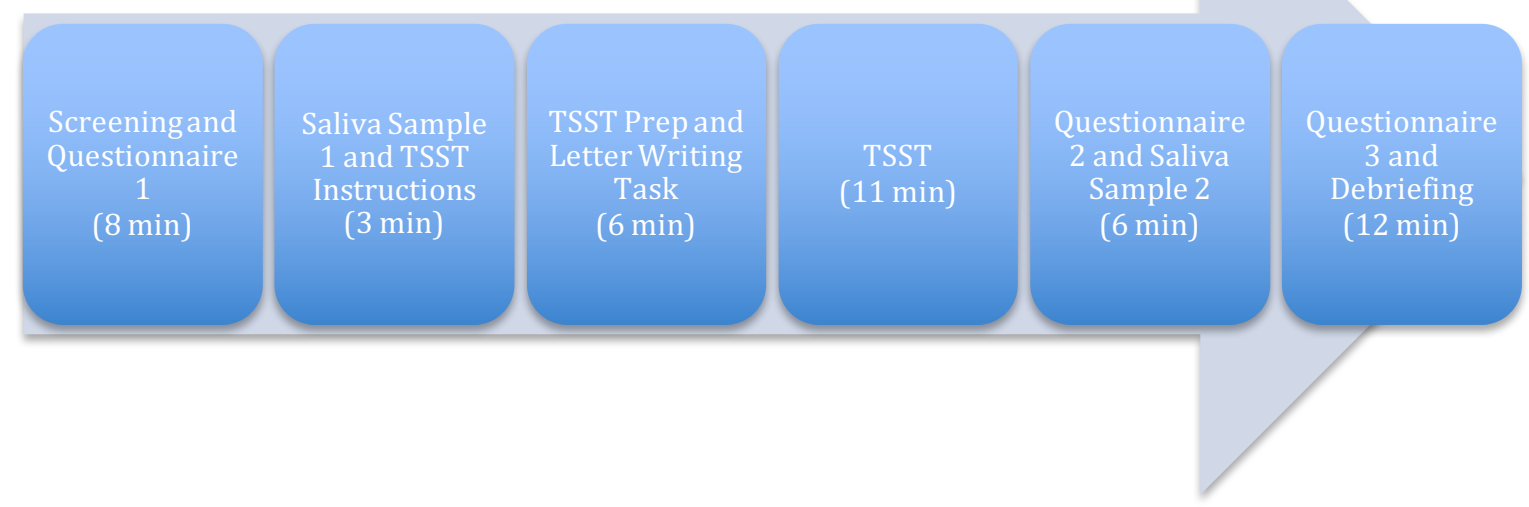

Figure 2. Flowchart of study procedure. Experimental testing lasted between 45 minutes to an hour depending on the speed of questionnaire completion. 


\section{Results}

\section{Culture}

Manipulation check. A manipulation check was performed to ensure there was an existing cultural difference between the two self-identified cultural groups, using an independent samples t-test. There was a significant difference between Asians and Asian Americans $(M=3.87, S D=.39)$ and European Americans $(M=3.65, S D=.51)$ on cultural values as scored by the AAVS-M, $t(93)=-2.44, p=.02, d=.48$, such that Asian and Asian American participants scored significantly higher on the Asian values scale.

Culture and different social support sources on cortisol reactivity. First, participants were included in final analyses only if they adhered to and completed the letter writing task. Second, an independent samples t-test and a one-way ANOVA were conducted to ensure there were no pre-existing differences in baseline cortisol between the groups. It was confirmed that there was no significant difference in baseline cortisol between Asian and Asian Americans $(M=.20 \mu \mathrm{g} / \mathrm{dL}, S D=.17)$ and European Americans $(M=.19 \mu \mathrm{g} / \mathrm{dL}, S D=.10), t(95)=-.36, p=.72$. Additionally, there were also no significant baseline cortisol differences between the control $(M=.22 \mu \mathrm{g} / \mathrm{dL}, S D=.16)$, parent $(M=.17 \mu \mathrm{g} / \mathrm{dL}, S D=.10)$, and peer $(M=.20 \mu \mathrm{g} / \mathrm{dL}, S D=.14)$ social support groups, $F(2,92)=.96, p=.39, \eta^{2}=.02$. Stress reactivity was calculated by subtracting baseline cortisol concentration from post-stressor concentration; therefore, a higher $\Delta$ Cortisol indicates a stronger stress response.

To test Hypotheses 1, 2, and 4, I performed a 2 (culture: Asians/Asian Americans and European Americans) x 3 (social support source: parent, peer, and control) analysis of 
covariance (ANCOVA). Biological sex was the covariate to control for potential sex differences in cortisol reactivity, as males have been reported to demonstrate higher cortisol reactivity as compared to females following exposure to a stressor (e.g., Kudielka \& Kirschbaum, 2005). See Table 1 for a summary of the results. Apart from a significant main effect from the covariate, sex, $F(1,88)=6.92, p=.01, \eta^{2}=.07$, there were no significant interaction, $F(2,88)=1.38, p=.26, \eta^{2}=.03$, nor significant main effects. Specifically, there were no significant main effects for culture, $F(1,88)=2.23$, $p=.14, \eta^{2}=.03$, or social support source, $F(1,88)=.32, p=.73, \eta^{2}=.01$. As the nonsignificant main effect revealed that there was no significant difference between the control group and the social support groups, Hypothesis 4 was not supported.

Table 1

Culture x Social Support ANCOVA for Cortisol Reactivity

\begin{tabular}{lcccc}
\hline Source & $d f$ & $F$ & $\eta^{2}$ & $p$ \\
\hline Sex & 1 & 6.92 & .073 & .010 \\
Social Support & 2 & .32 & .007 & .725 \\
Culture & 1 & 2.24 & .025 & .138 \\
$\begin{array}{l}\text { Social } \\
\text { Support*Culture }\end{array}$ & 2 & 2.38 & .030 & .256 \\
Error & 88 & & & \\
\hline
\end{tabular}

It should be noted, however, that the $\Delta$ Cortisol difference between the parent $(M=.08 \mu \mathrm{g} / \mathrm{dL}, S D=.18)$ and peer $(M=.20 \mu \mathrm{g} / \mathrm{dL}, S D=.20)$ groups among European American participants approached significance and had a medium effect size, $p=.06, d=$ .63. Similar findings were discovered with the $\Delta$ Cortisol difference between European American and Asian/Asian American participants assigned to the parent social support 
condition, $t(28)=-1.83, p=.08, d=.64$. Although they were not statistically significant, these results trended towards a direction that was consistent with Hypothesis 2. Figure 3 illustrates these differences.

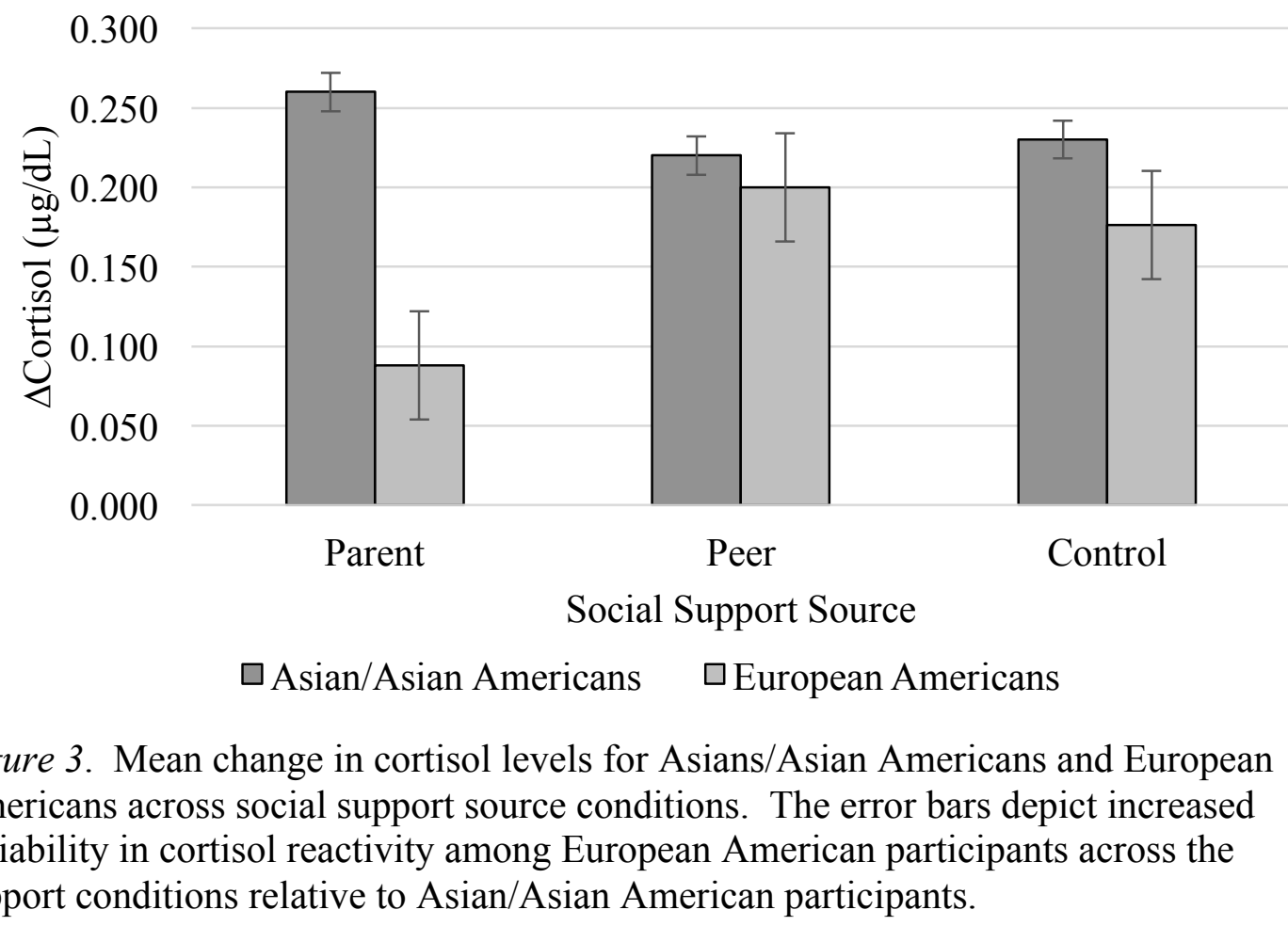

To examine the difference in effectiveness of explicit support between cultures for Hypothesis 3, I ran an ANCOVA using only participants who were assigned to a support condition. The two social support sources were collapsed into one variable to represent explicit support. Sex was again included as the covariate. No significant cortisol differences were found between Asians and Asian Americans $(M=.23 \mu \mathrm{g} / \mathrm{dL}, S D=.32)$ and European Americans $(M=.14 \mu \mathrm{g} / \mathrm{dL}, S D=.20)$ regarding their response to receiving explicit social support prior to a psychosocial stressor, $F(1,58)=1.94, p=.17, \eta^{2}=.03$. 
These results showed that Hypothesis 3 was not supported. The covariate (sex) was, however, significant, $F(1,58)=5.56, p=.02, \eta^{2}=.09$, such that males $(M=.28 \mu \mathrm{g} / \mathrm{dL}$, $S D=.26)$ had higher stress reactivity following the stressor than females $(M=.13 \mu \mathrm{g} / \mathrm{dL}$, $S D=.25)$

Immigrant generation. As generation may influence the enculturation of values, analyses were conducted to examine the potential effect of immigrant generation. The few Asian American participants who reported being third generation $(n=4)$ were excluded, as the sample size was too small to apply to all conditions. Third generation participants were not combined with second generation participants, due to past findings that indicated that the enculturation of Asian values in Asian Americans may disappear by the third generation (Heine, Lehman, Markus, \& Kitayama, 1999; Taylor et al., 2004).

Within Asian and Asian American participants, I performed a 2 (generation: first and second) $x 3$ (social support source) ANCOVA using sex as the covariate. As summarized in Table 2, results revealed no significant interaction between generation and social support within Asians and Asian Americans, $F(2,34)=1.37, p=.27, \eta^{2}=.08$. There were also no significant main effects for generation, $F(1,34)=.24, p=.63, \eta^{2}=.01$, or social support, $F(1,34)=.23, p=.80, \eta^{2}=.01$. 
Table 2

Immigrant Generation x Social Support ANCOVA for Cortisol Reactivity

\begin{tabular}{lcccc}
\hline Source & $d f$ & $F$ & $\eta^{2}$ & $p$ \\
\hline Sex & 1 & 1.47 & .041 & .234 \\
Social Support & 2 & .23 & .013 & .798 \\
Generation & 1 & .24 & .007 & .627 \\
Social Support* & & & & .268 \\
Generation & 2 & 1.37 & .075 & \\
Error & 34 & & & \\
\hline
\end{tabular}

For European Americans, sample sizes for first $(n=4)$ and second $(n=7)$ generations were too small for within culture comparisons. Instead, I re-ran the 2 (culture) x 3 (social support) ANCOVA with only the second and third+ generations of European Americans and also excluded the few third-generation Asian American participants to ensure enculturation of individualistic and collectivism values within the respective group. Still, there was no significant interaction, $F(2,73)=.97, p=.39, \eta^{2}=.03$. There were also no significant main effects for culture, $F(1,73)=2.27, p=.14, \eta^{2}=.03$, or social support, $F$ $(1,73)=.10, p=.91, \eta^{2}=.003$

Culture and support type. As there are no current studies examining the potential interaction between support type (i.e., emotional and informational) and culture on social support effectiveness, I conducted an additional, exploratory analysis to study the topic. I performed a 2 (culture) x 4 (support type: emotional, informational, both, and no support) ANCOVA with sex as the covariate. There was no significant interaction effect between culture and support type, $F(3,86)=.85, p=.47, \eta^{2}=.03$. The main effect for culture was once again non-significant, as was the main effect for support type, $F(3,83)=.75, p$ 
$=.54, \eta^{2}=.03$. The covariate, sex, was again significant, $F(1,86)=6.88, p=.01, \eta^{2}=$ .07. Additionally, a chi-square test of independence was conducted to explore the differences in distribution of support type use (excluding no support) between Asians/Asian Americans and European Americans. These distributions can be seen below in Figure 4. Results revealed no significant differences in the distribution of support type use, $X^{2}=(2, N=61), p=.09$.

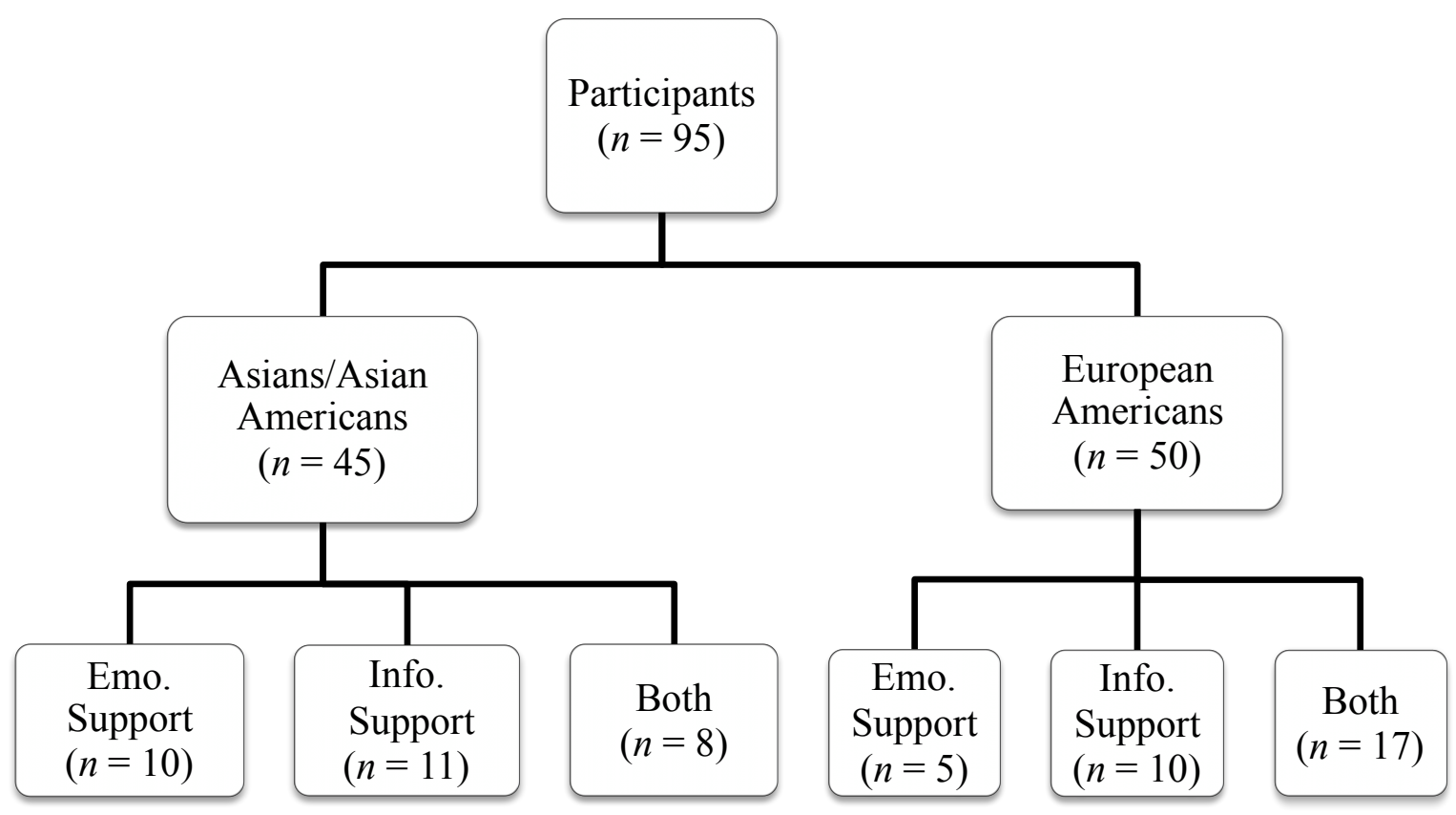

Figure 4. Preference of support type according to Asians/Asian Americans and European Americans. The remaining participants not shown in the chart were control participants ( $n=16$ for Asians/Asian Americans and $n=18$ for European Americans).

\section{Gender Role Orientation}

Gender roles and social support sources on cortisol reactivity. Again, I first confirmed there were no significant baseline cortisol differences for two variables: gender roles, $F(3,91)=.80, p=.50, \eta^{2}=.03$, and sex, $t(93)=-.10, p=.93$. In the 
interest of more broadly examining the data, I ran a 3 (social support) x 4 (gender role) ANOVA. An ANOVA rather than an ANCOVA with sex as the covariate was conducted here due to the violation of the independence assumption required for performing an ANCOVA, specifically between gender roles and sex. A three-way ANOVA with sex as the third factor was attempted, but there were issues with sample size following the attrition and omission of some participants. Figure 5 below summarizes the breakdown of gender roles and sex within this sample.

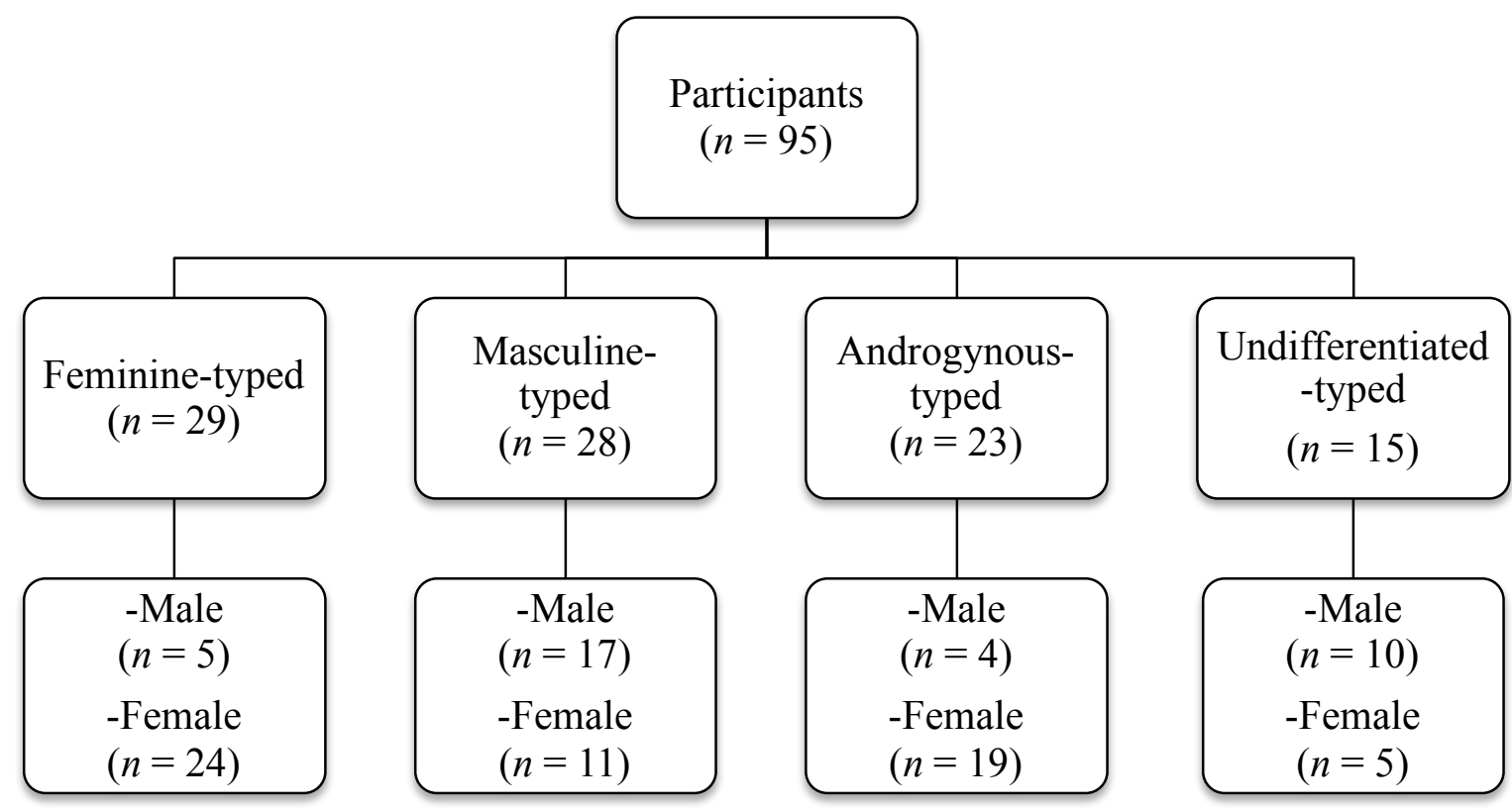

Figure 5. Frequencies of gender orientations within this sample.

There was a significant main effect for gender roles, $F(3,83)=2.84, p=.04, \eta^{2}=.09$, but there was no significant main effect for social support, $F(2,83)=.19, p=.83, \eta^{2}=$ .01. There was also no significant interaction to show support for Hypothesis $5, F(6,83)$ $=.78, p=.59, \eta^{2}=.05$. Pairwise comparisons were used to further assess the differences 
in cortisol reactivity across the gender roles. Here, I expected to find lower cortisol reactivity for androgynous individuals as mentioned in Hypothesis 6; however, analyses showed a significant difference in $\Delta$ Cortisol between feminine $(M=.11 \mu \mathrm{g} / \mathrm{dL}, S D=.19)$ and undifferentiated individuals $(M=.33 \mu \mathrm{g} / \mathrm{dL}, S D=.38), p=.04$, such that undifferentiated individuals had a larger stress response following the TSST as compared to feminine individuals. Because I was not interested in social support source in relation to gender roles, I then collapsed the two source conditions into one generalized social support condition and compared it to the control group that did not receive support. As seen in Table 3 , results from a $2 \times 4$ ANOVA found no significant interaction, $F(3,87)=$ $.64, p=.60, \eta^{2}=.02$, and no significant main effect for social support, $F(1,87)=.04, p$ $=.85, \eta^{2}=.00$, or gender roles, $F(3,87)=1.88, p=.14, \eta^{2}=.06$.

Table 3

Social Support x Gender Roles ANOVA for Cortisol Reactivity

\begin{tabular}{lcccc}
\hline Source & $d f$ & $F$ & $\eta^{2}$ & $p$ \\
\hline Social Support & 1 & .037 & .000 & .847 \\
Gender Role & 3 & 1.878 & .061 & .139 \\
$\begin{array}{l}\text { Social Support*Gender } \\
\text { Role }\end{array}$ & 3 & .635 & .021 & .595 \\
Error & 87 & & & \\
\hline
\end{tabular}

Though the significant differences in $\Delta$ Cortisol between undifferentiated and feminine individuals disappeared with the collapsing of the two social support groups into one, the $\Delta$ Cortisol differences between gender roles illustrated in Figure 6 may be suggestive, and further assessment of gender roles' impact on stress with an increased sample size may be warranted. 


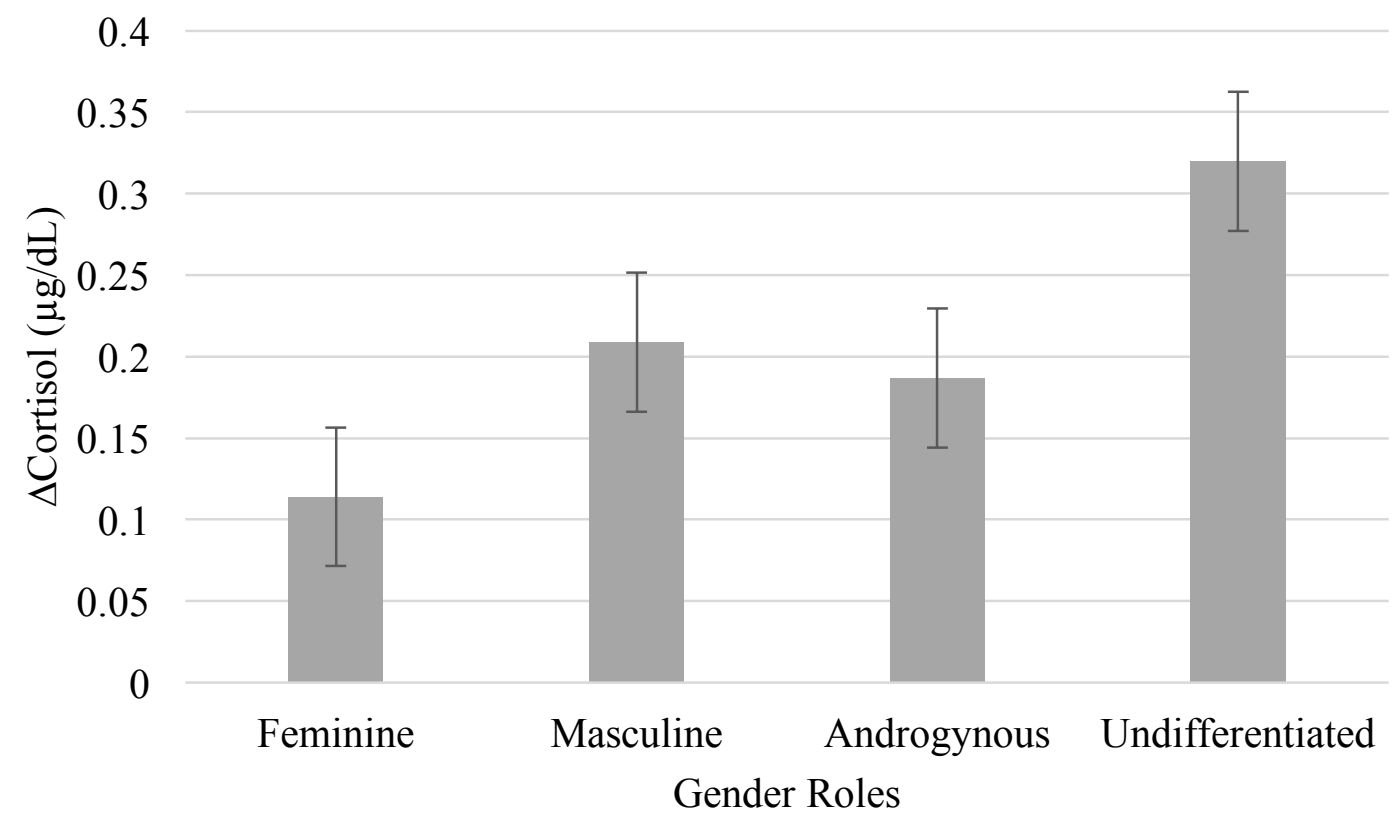

Figure 6. Mean change in cortisol levels across the four gender role orientations. The error bars depict similar variations in data for cortisol reactivity across the gender roles.

Further, there was an attempt to conduct a 2 (culture) x 3 (social support) $\mathrm{x} 4$ (gender role) three-way ANOVA, but the relatively small sample due to the attrition and omission of participants as a result of noncompliance issues, prohibited such analyses. As seen earlier in Figure 1, the original sample size of 144 was reduced to a total of 95 participants for the final analyses. And as a result, in one instance, none of the remaining male Asian participants scored as feminine on the BSRI. In sum, these analyses did not show support for Hypotheses 5 and 6.

Gender and social support type. Knowing from past literature that social support type may influence support effectiveness differently between gender roles, the use of emotional and/or informational support had to be considered. The following analysis was 
conducted to test Hypothesis 7, as well as to explore the relationship between support type and the non-binary gender orientations. As with previous gender role x social support analyses, another ANOVA was conducted, rather than ANCOVA, to prevent violating the assumption of independence between sex (covariate) and gender roles. The 4 (support type: emotional, informational, both, and control) x 4 (gender role) ANOVA revealed no significant interaction between the two factors, $F(9,79)=.60, p=.79, \eta^{2}=$ .06 , and therefore, did not show support for Hypothesis 7 . Like the analyses before, the main effect for support type was not significant, but the main effect for gender roles was significant, $F(1,79)=4.05, p=.01, \eta^{2}=.13$. As seen previously in the 3 (social support) $\mathrm{x} 4$ (gender role) ANOVA, pairwise analyses revealed a significant difference in $\Delta$ Cortisol between undifferentiated and feminine individuals, such that undifferentiated individuals, as compared to feminine individuals, had a larger stress response following the stressor. However, there was the issue of sample size again here. Because support type and gender role identity were variables I could not control for, and because of the experienced attrition and omission of 49 participants, the sample sizes for some conditions were as small as two individuals. 


\section{Discussion}

Extending previous findings, the present study examined the role of culture and gender roles in social support-seeking behaviors and stress outcomes. Building on previous research that established cultural differences in self-reported preferences for social support source (Wang et al., 2010; Wang \& Lau, 2015), I used an experimental manipulation of stress and support source to further study this difference. Because prior studies neglected to consider the role of non-binary gender orientations in social supportseeking behaviors and outcomes, I addressed this gap by researching the interaction between four gender roles and social support on stress. I also expanded on social support by assessing type, specifically emotional and informational types of support in relation to both culture and gender.

First, I examined differences in cortisol reactivity between and within Asians/Asian Americans and European Americans, following a psychosocial stressor and seeking social support from one of two sources (i.e., support from a parent or a peer). For Hypothesis 1, I predicted that Asians and Asian Americans would benefit more from social support when seeking it from a peer as compared to a parent, which is consistent with the collectivist mindset. As documented in past research, Asians and Asian Americans more often seek advice from peers so as to avoid any possible strain on relational ties and to maintain familial harmony (Wang \& Lau, 2015; Wang et al., 2010). For European Americans, early research posited that social support would be beneficial regardless of source because their individualistic values prioritize the needs of the individual (Markus \& Kitayama, 1991), which in this case would simply be obtaining 
support. However, recent research reports that European Americans more frequently seek advice from their parents over their peers (Wang \& Lau, 2015; Wang et al., 2010). This led me to hypothesize that they would also benefit more from seeking advice from a parent as compared to a peer, which would be seen as lower cortisol reactivity and reduced stress (Hypothesis 2). A letter-writing task was used to evoke explicit social support, and consistent with reports that implicit as opposed to explicit social support was more beneficial for Asians and Asian Americans (Taylor et al. 2007), I hypothesized that Asians and Asian Americans would generally benefit less from the support task than European Americans (Hypothesis 3). Despite the diminished beneficial qualities of explicit support in Asians and Asian Americans, I predicted that any social support would still be, overall, more beneficial than no support at all (Hypothesis 4).

Although the current findings are suggestive, they did not solidly support the hypotheses for culture's influence on the benefit of social support from differing sources. More specifically, there was a marginal but not statistically significant difference in cortisol reactivity among European Americans assigned to the parent versus peer social support conditions. In other words, European American participants appeared to benefit more from the parental support condition, as seen as reduced cortisol levels following the psychosocial stressor, relative to those observed with the peer support seeking condition. This is consistent with the direction set by Hypothesis 2 .

It is also worth noting that research findings on this topic appear to be mixed, with one recent report of increased parent support for European Americans, but not Asians and Asian Americans, during a period of transitioning into young adulthood (Guan \& Fuligni, 
2016), and another report of Asian natives receiving more support from parents relative to European Americans (Fingerman et al., 2016). Despite the contrasting findings, Asian participants in the latter study also reported that they were less satisfied with the support received from their parents. This points out a potentially important factor when considering the stress-mitigating effects of social support, and that is the distinction between support sought and the quality of support received. Perhaps receiving unsatisfactory advice, regardless of quantity, is not beneficial, and it may motivate individuals to seek future advice elsewhere.

Another marginal but not statistically significant difference found in the present study was for cortisol reactivity between European Americans and Asians/Asian Americans assigned to the parent condition, with Asians and Asian Americans having a greater increase in cortisol. This finding may also warrant further exploration as it relates to the basis of Hypotheses 1 and 2, and is in line with findings that parent support was less satisfactory for Asian nationals. This could also mean that European Americans, in comparison to Asians and Asian Americans, find parental social support more helpful. However, inconsistent with Hypothesis 1, Asians and Asian Americans did not benefit more from seeking social support from peers. It could be that the explicit nature of the writing task diminished the beneficial qualities of support, which is related to Hypothesis 3. However, when testing Hypothesis 3, there were no significant differences in cortisol reactivity between Asians/Asian Americans and European Americans regarding the effectiveness of explicit social support. It may be that writing a letter to seek support does not evoke the feeling of explicit support as strongly as asking the source directly. 
Perhaps a mention of sending the letter to the recipient would evoke stronger emotions of support seeking.

Moreover, as social support is believed to generally provide beneficial effects despite the presence of confounding variables, it was also expected that participants in either of the social support groups would have lower $\Delta$ Cortisol than those in the no support group, as expected in Hypothesis 4. However, there were also no significant differences for stress reactivity between the control group and the social support groups. A similar result was found in a study by Taylor and colleagues (2007), which utilized a comparable writing task to evoke social support. Participants assigned to the control group (i.e., no social support) showed few signs of stress following a psychosocial stressor. The authors proposed that their control task could have unintentionally acted as a distraction from the psychosocial stressor, thus decreasing stress. With that considered, the task was altered for the current study with the aim of focusing the participants on their worries and concerns in anticipation for the public speech task. However, it is possible that the act of writing down their worries and concerns prior to facing them may be cathartic for some individuals. Future studies may benefit from more in-depth examinations of the social support letter writing task.

Though this sample's Asian and Asian American participants had more Asian cultural values than the European American participants, further tests were conducted to determine the influence of immigrant generation by only including Asian and Asian American participants of first or second generation and European American participants of second or third+ generation. The basis for restriction criteria stemmed from previous 
culture and social support studies (e.g., Wang \& Lau, 2015; Wang et al., 2010). The results were again not significant for cortisol reactivity differences across social support groups with the generations trimmed down. Analyses of AAVS-M scores within both culture groups also showed no differences in scores between generations, though that may be influenced by small sample sizes for certain generations. Still, it might be useful to consider immigrant generations during the development of future studies, as studies have concluded that any social support differences may minimalize or disappear by the third generation and beyond for Asian Americans (Heine, Lehman, Markus, \& Kitayama, 1999; Taylor et al., 2004).

Lastly, I explored the interaction of culture and social support type with no previous studies on the two variables to elaborate on. The analyses did not reveal a significant interaction between the three support types (i.e., emotional, informational, and both combined) and culture, and a chi-square test of independence also found no significant differences in distribution of support type usage between the two cultures. This may indicate that there were no preferences for different support types between the European Americans and Asians/Asian Americans in our sample.

While previous research on the influence of biological sex and gender roles on social support has established differences in support-seeking and receiving behaviors and outcomes, it is also important to explore beyond binary categorization of gender. As the concept of gender becomes more fluid and complex, research must accommodate to this growth by reaching these emerging populations and subsequently improving treatment and counseling so that the methods used are appropriate for all types of individuals. This 
prompted me to further explore my second main variable of interest, gender roles, but with the addition of androgynous and undifferentiated individuals, as proposed by Bem (1974a) and Spence et al. (1977). Though the field of social support literature lacks studies examining all four gender roles, various other studies revealed differences in adjustment and self-esteem across the gender roles that prompted my hypotheses. One study even established the increased use of unhealthy coping techniques in undifferentiated individuals as compared to the other gender orientations (Fugitt et al., 2017).

Particularly, for Hypothesis 5, I predicted that feminine individuals would benefit more from seeking social support (regardless of source) as compared to masculine individuals, as well as individuals who received no social support. As undifferentiated individuals have been found to more likely use unhealthy coping techniques, I also hypothesized that they would have a higher cortisol response after receiving social support relative to feminine individuals. For Hypothesis 6, I predicted that androgynous individuals, due to their high psychological health, would have a lower stress response following the TSST relative to the other gender roles. Continuing further, because past literature has demonstrated that social support type (i.e., emotional, informational, or instrumental) affects the gender roles differently, I also assessed support type as a variable in interaction with gender roles. For Hypothesis 7, it was expected that emotional and informational support would most benefit feminine and masculine individuals, respectively. Analyses for the relationship between support type and the 
remaining two gender role orientations, androgynous and undifferentiated, were more exploratory in nature due to a lack of previous research.

Hypothesis 5 was not supported by the findings in the current study, as seen by the non-significant interaction between gender roles and social support. This could be due to the lack of manipulation for the social support types utilized in the writing task. Specifically, rather than being assigned to a group, participants had the option to seek emotional and/or informational support. Perhaps the participants utilized the types of support they were most comfortable with, which resulted in a lack of difference in cortisol reactivity here, as well as for Hypothesis 7. This lack of significant findings may also in part be due to a reduced sample size following attrition and omission of participants with incomplete data. Because some of the conditions included only two participants, it was difficult to comment on the distribution of support type use across the gender roles.

Although there were no significant differences in $\Delta$ Cortisol across the gender orientations in response to social support to support my predictions, the explorative analyses conducted may indicate that undifferentiated individuals may have higher increases in cortisol following stressors in comparison to feminine individuals. Perhaps undifferentiated individuals, being low on both feminine and masculine traits, were not equipped with suitable techniques needed to cope with the stress task. However, the caveats here were that this finding was not replicated in a second ANOVA that involved combining two social support groups into one, and that I was also unable to control for biological sex. As sex may be closely related to gender orientation, I cannot fully state 
that these gender role effects are independent of sex. As to why androgynous individuals did not display a lower stress response relative to the other orientations, as predicted in Hypothesis 6 , it could be explained by their composition of traits. Specifically, because they share traits with masculine and feminine individuals in high and equal quantity, maybe a mixture of those traits (both helpful and unhelpful in handling stress) was called on during the TSST, and it was not enough to significantly set their coping abilities apart from the other orientations.

\section{Limitations and Strengths}

As with other studies, there were a few challenges and limitations experienced while running this experiment, which must be discussed to improve future research. For one, the letter writing task that was utilized to evoke social support came with some unforeseen difficulties. Ideally, on the day of the study, participants would have brought with them a parent or a peer to act as their social support source to ensure social support was provided. However, due to constraints of potential scheduling difficulties and the source's distance from campus, this method would have been highly impractical. With the letter writing task utilized in previous studies, it was an appropriate choice to borrow and adapt to fit the current interests of this study and to control for potential confounds.

Moreover, while it was specified in the prompt that the participant should both write to their social support source asking for advice and consider what advice their source would respond with, perhaps the task could be simplified, as participants are likely flustered by the prospect of delivering a speech following the task. Participants were also informed that their letter would be checked for proper completion as an incentive for the 
participants to follow and complete the prompt, but it was noted that some participants did not use the entire allotted three minutes to work on their task. This could have possibly resulted in extra speech practicing time. Future studies may want to consider other options to encourage students to write for the entire time allotted. Additionally, no manipulation check has been cited for these writing tasks, which makes it difficult to confirm the validity of the task in evoking social support. A revision to ask participants whether they found the writing task to be supportive may be required.

In relation to the writing task, there is also the possibility that the type of social support sought may influence its efficacy. Specifically, though participants were prompted to ask for explicit advice regarding the upcoming speech, they could have potentially asked for emotional and/or informational support. While support type was assessed in connection to culture and gender roles, and there were no significant associations, it may warrant further examination with an increased sample size and more control over the manipulation of support type. It may also be that the nature of the TSST conflicts with the differing benefits of support type. Recall from earlier that one of the requirements of successful social support is that the type of support used must match the required resources elicited by the stressor. This means that whichever support types are required by the public speech task may clash with the preferred support type used by the individual.

Further, the lack of a pre-existing guideline for coding support letters as emotional or informational also allowed for some potential subjectivity to slip into the coding process. 
Studies should consider either developing a rubric for analyzing support letter content or randomly assigning participants to specific support type conditions.

Another consideration for future research concerns the final sample size of this study. An a prior power analysis was conducted at the start of the study using $G^{*}$ Power (Erdfelder, Lang, \& Buchner, 2007), indicating a sample size of 158 participants was needed to achieve adequate power. A total of 144 participants were recruited, but 49 were omitted due to attrition and failure to comply to certain aspects of the study. With more participants included in the final analyses, I may have been able to uncover results that are not possible at this current state. Lastly, because the primary aim here was to examine social support's physiological effects, there was no scale for the self-reported measure of mood included in the study. In hindsight, the addition of such an inventory would have made an interesting comparison between mental and physiological stress in reaction to social support.

Despite these limitations, this study is not without its notable strengths. There were various measures taken to ensure the accuracy of data collected for this study. First, researchers and lab assistants were trained to memorize the same scripts and protocols to prevent any confounds related to subjectivity during the experiment process. Second, biological assays of the saliva samples were also conducted strictly following protocol to ensure the best reading for each sample. This study also explored topics sparsely examined before in the field of social support, including but not limited to the inclusion of non-binary gender orientations, and the potential relationship between culture and usage of different social support types. Finally, there were attempts to improve issues 
previously found with the letter task used to manipulate social support. While these changes were not entirely successful, they still provide additional insight for future studies utilizing similar tasks.

\section{Conclusion}

In summary, the present study set out to examine the physiological differences between Asians/Asian Americans and European Americans regarding preference for seeking social support from parents or peers, as well as to further examine the role of gender role orientation in the realm of social support. Though no significant differences were found between cultures for their physiological reaction to both explicit support and parent versus peer social support, some marginal differences were demonstrated in line with my hypotheses. The results suggested that European Americans may physiologically respond to social support in a more beneficial manner when the support is sought from a parent than from a peer, and that, in comparison to Asians and Asian Americans, they may benefit more from parent support. As with culture, the results for gender roles also did not support my hypotheses. For one, it was found that, relative to feminine individuals, undifferentiated individuals had higher cortisol reactivity following the stressor. This finding was consistent with previous research on gender roles and coping. However, sex was a variable I was unable to disentangle from gender roles, so sex's influence on those results cannot be completely ruled out. Additionally, previous findings that demonstrated that emotional and informational support were more beneficial to feminine and masculine individuals, respectively were not replicated here. With further research and a larger sample size, interactions between the four gender roles and 
the other variables could potentially be unveiled. Although I did not discover all that I set out to, these findings may still prompt counseling and treatment programs to consider parent and peer dynamics in relation to the client's cultural background, as well as the client's gender orientation when discussing social support. 


\section{References}

Adams, G., \& Plaut, V. C. (2003). The cultural grounding of personal relationship: Friendship in North American and West African worlds. Personal Relationships, 10(3), 333-347.

Aneshensel, C. S., \& Stone, J. D. (1982). Stress and depression: A test of the buffering model of social support. Archives of General Psychiatry, 39(12), 1392.

Barbee, A. P., Cunningham, M. R., Winstead, B. A., Derlega, V. J., Gulley, M. R., Yankeelov, P. A., \& Druen, P. B. (1993). Effects of gender role expectations on the social support process. Journal of Social Issues, 49(3), 175-190.

Bem, S. L. (1974a). The measurement of psychological androgyny. Journal of Consulting and Clinical Psychology, 42(2), 155.

Bem, S. L. (1974b). Bem Sex Role Inventory. Psyctests, doi:10.1037/t00748-000

Bem, S. L. (1977). On the utility of alternative procedures for assessing psychological androgyny. Journal of Consulting and Clinical Psychology, 45(2), 196.

Bem, S. L., Martyna, W., \& Watson, C. (1976). Sex typing and androgyny: Further explorations of the expressive domain. Journal of Personality and Social Psychology, 34(5), 1016.

Berger, B. G. (1994). Coping with stress: The effectiveness of exercise and other techniques. Quest, 46(1), 100-119.

Berkman, L. F., \& Syme, S. L. (1979). Social networks, host resistance, and mortality: a nine-year follow-up study of Alameda County residents. American Journal of Epidemiology, 109(2), 186-204.

Bryant, R. A., Harvey, A. G., Dang, S. T., Sackville, T., \& Basten, C. (1998). Treatment of acute stress disorder: a comparison of cognitive-behavioral therapy and supportive counseling. Journal of Consulting and Clinical Psychology, 66(5), 862.

Carver, C. S. (1997). You want to measure coping but your protocol's too long: Consider the Brief COPE. International Journal of Behavioral Medicine, 4, 92-100

Cobb, S. (1976). Social support as a moderator of life stress. Psychosomatic Medicine, 38(5), 300-314. 
Cohen, S., \& Wills, T. A. (1985). Stress, social support, and the buffering hypothesis. Psychological Bulletin, 98(2), 310-357. doi:10.1037/0033-2909.98.2.310

Collins, N. L., \& Miller, L. C. (1994). Self-disclosure and liking: A meta-analytic review. Psychological Bulletin, 116, 457-475.

Copeland, E. P., \& Hess, R. S. (1995). Differences in young adolescents' coping strategies based on gender and ethnicity. The Journal of Early Adolescence, 15(2), 203-219.

Coyne, J. C., \& Downey, G. (1991). Social factors and psychopathology: Stress, social support, and coping processes. Annual Review of Psychology, 42(1), 401-425.

Dunn, J., Bretherton, I., \& Munn, P. (1987). Conversations about feeling states between mothers and their young children. Developmental Psychology, 23(1), 132.

Edwards, C. P. (1993). Behavioral sex differences in children of diverse cultures: The case of nurturance to infants. In M. E. Pereira \& L. A. Fairbanks (Eds.), Juvenile primates: Life History, Development, and Behavior (pp. 327-338). New York: Oxford University Press.

Faul, F., Erdfelder, E., Lang, A.-G., \& Buchner, A. (2007). G*Power 3: A flexible statistical power analysis program for the social, behavioral, and biomedical sciences. Behavior Research Methods, 39, 175-191.

Fingerman, K. L., Cheng, Y. P., Kim, K., Fung, H. H., Han, G., Lang, F. R., ... \& Wagner, J. (2016). Parental involvement with college students in Germany, Hong Kong, Korea, and the United States. Journal of Family Issues, 37(10), 1384-1411.

Fugitt, J. L., Ham, L. S., \& Bridges, A. J. (2017). Undifferentiated gender role orientation, drinking motives, and increased alcohol use in men and women. Substance Use \& Misuse, 52(6), 760-772.

Glaser, R., \& Kiecolt-Glaser, J. K. (2005). Science and society: Stress-induced immune dysfunction: implications for health. Nature Reviews Immunology, 5(3), 243-251. doi: $10.1038 /$ nri1571

Goodenow, C., Reisine, S. T., \& Grady, K. E. (1990). Quality of social support and associated social and psychological functioning in women with rheumatoid arthritis. Health Psychology, 9(3), 266. 
Grossman, M., \& Wood, W. (1993). Sex differences in intensity of emotional experience: A social role interpretation. Journal of Personality and Social Psychology, 65, $1010-1022$.

Guan, S. S. A., \& Fuligni, A. J. (2016). Changes in parent, sibling, and peer support during the transition to young adulthood. Journal of Research on Adolescence, 26(2), 286-299.

Gump, B. B., Polk, D. E., Kamarck, T. W., \& Shiffman, S. M. (2001). Partner interactions are associated with reduced blood pressure in the natural environment: Ambulatory monitoring evidence from a healthy, multiethnic adult sample. Psychosomatic Medicine, 63(3), 423-433.

Hefner, J., \& Eisenberg, D. (2009). Social support and mental health among college students. American Journal of Orthopsychiatry, 79(4), 491-499.

Heine, S. J., Lehman, D. R., Markus, H. R., \& Kitayama, S. (1999). Is there a universal need for positive self-regard?. Psychological Review, 106(4), 766.

Heinrichs, M., Baumgartner, T., Kirschbaum, C., \& Ehlert, U. (2003). Social support and oxytocin interact to suppress cortisol and subjective responses to psychosocial stress. Biological Psychiatry, 54(12), 1389-1398. doi:10.1016/S00063223(03)00465-7

House, J. S., Landis, K. R., \& Umberson, D. (1988). Social relationships and health. Science, 241(4865), 540-545.

Kagan, J. (1964). Acquisition and significance of sex typing and sex role identity. Review of Child Development Research, 1, 137-167.

Kaslow, N. J., Deering, C. G., \& Racusin, G. R. (1994). Depressed children and their families. Clinical Psychology Review, 14(1), 39-59.

Kim, H. S. (2002). We talk, therefore we think? A cultural analysis of the effect of talking on thinking. Journal of Personality and Social Psychology, 83(4), 828.

Kim, B. K., Li, L. C., \& Ng, G. F. (2005). The Asian American values scale-multidimensional: development, reliability, and validity. Cultural Diversity and Ethnic Minority Psychology, 11(3), 187.

Kim, B. K., Li, L. C., \& Ng, G. F. (2005). Asian American Values ScaleMultidimensional. Psyctests, doi:10.1037/t00037-000 
Kim, H. S., Sherman, D. K., Ko, D., \& Taylor, S. E. (2006). Pursuit of Comfort and Pursuit of Harmony: Culture, Relationships, and Social Support Seeking. Personality and Social Psychology Bulletin, 32(12), 1595-1607. doi:10.1177/0146167206291991

Kim, H. S., \& Sherman, D. K. (2007). " Express yourself": culture and the effect of selfexpression on choice. Journal of Personality and Social Psychology, 92(1), 1.

Kirschbaum, C., Pirke, K. M., \& Hellhammer, D. H. (1993). The 'Trier Social Stress Test'-a tool for investigating psychobiological stress responses in a laboratory setting. Neuropsychobiology, 28(1-2), 76-81.

Kirschbaum, C., \& Hellhammer, D. H. (1994). Salivary cortisol in psychoneuroendocrine research: recent developments and applications. Psychoneuroendocrinology, 19(4), 313-333.

Kudielka, B. M., Hellhammer, D. H., Kirschbaum, C., Harmon-Jones, E., \& Winkielman, P. (2007). Ten years of research with the Trier Social Stress Test-revisited. Social Neuroscience: Integrating Biological and Psychological Explanations of Social Behavior, 56-83.

Kudielka, B. M., \& Kirschbaum, C. (2005). Sex differences in HPA axis responses to stress: a review. Biological Psychology, 69(1), 113-132.

Kudielka, B. M., Schommer, N. C., Hellhammer, D. H., \& Kirschbaum, C. (2004). Acute HPA axis responses, heart rate, and mood changes to psychosocial stress (TSST) in humans at different times of day. Psychoneuroendocrinology, 29(8), 983-992.

Larzelere, M. M., \& Jones, G. N. (2008). Stress and health. Primary Care: Clinics in Office Practice, 35(4), 839-856.

Lepore, S. J., Allen, K. A., \& Evans, G. W. (1993). Social support lowers cardiovascular reactivity to an acute stressor. Psychosomatic Medicine, 55(6), 518-524.

Lutgendorf, S. K., Sood, A. K., Anderson, B., McGinn, S., Maiseri, H., Dao, M., ... \& Lubaroff, D. M. (2005). Social support, psychological distress, and natural killer cell activity in ovarian cancer. Journal of Clinical Oncology, 23(28), 7105-7113.

Malatesta, C. Z., Culver, C., Tesman, J. R., \& Shepard, B. (1989). The development of emotion expression during the first two years of life. Monographs of the Society for Research in Child Development, 54(1/2), 1-104. 
Markus, H. R., \& Kitayama, S. (1991). Culture and the self: Implications for cognition, emotion, and motivation. Psychological Review, 98(2), 224.

Mason, J. W. (1968). A review of psychoendocrine research on the pituitary-adrenal cortical system. Psychosomatic Medicine, 30(5), 576-607.

Michaud, S. L., \& Warner, R. M. (1997). Gender differences in self-reported response to troubles talk. Sex Roles, 37(7-8), 527-540. doi:10.1023/A:1025606918913

Mojaverian, T., \& Kim, H. S. (2013). Interpreting a helping hand: Cultural variation in the effectiveness of solicited and unsolicited social support. Personality and Social Psychology Bulletin, 39(1), 88-99.

O'connor, K., Mann, D. W., \& Bardwick, J. M. (1978). Androgyny and self-esteem in the upper-middle class: a replication of Spence. Journal of Consulting and Clinical Psychology, 46(5), 1168.

Orlofsky, J. L., \& Windle, M. T. (1978). Sex-role orientation, behavioral adaptability and personal adjustment. Sex Roles, 4(6), 801-811.

Oxman, T. E., Berkman, L. F., Kasl, S., Freeman Jr, D. H., \& Barrett, J. (1992). Social support and depressive symptoms in the elderly. American Journal of Epidemiology, 135(4), 356-368.

Penninx, B. W., van Tilburg, T., Boeke, A. J. P., Deeg, D. J., Kriegsman, D. M., \& van Eijk, J. T. M. (1998). Effects of social support and personal coping resources on depressive symptoms: different for various chronic diseases?. Health Psychology, 17(6), 551.

Perloff, D., Sokolow, M., \& Cowan, R. (1983). The prognostic value of ambulatory blood pressures. Jama, 249(20), 2792-2798.

Ptacek, J. T., Smith, R. E., \& Zanas, J. (1992). Gender, appraisal, and coping: A longitudinal analysis. Journal of Personality, 60(4), 747-770.

Reevy, G. M., \& Maslach, C. (2001). Use of social support: gender and personality differences. Sex Roles, 44(7/8), 437-459.

Renk, K., \& Creasey, G. (2003). The relationship of gender, gender identity, and coping strategies in late adolescents. Journal of Adolescence, 26(2), 159-168. 
Spence, J. T., Helmreich, R., \& Stapp, J. (1975). Ratings of self and peers on sex role attributes and their relation to self-esteem and conceptions of masculinity and femininity. Journal of Personality and Social Psychology, 32(1), 29.

Taylor, S. E. (2007). Social support. Foundations of Health Psychology, 145171.

Taylor, S. E., Sherman, D. K., Kim, H. S., Jarcho, J., Takagi, K., \& Dunagan, M. S. (2004). Culture and Social Support: Who Seeks It and Why?. Journal of Personality and Social Psychology, 87(3), 354-362. doi:10.1037/00223514.87.3.354

Taylor, S. E., Welch, W. T., Kim, H. S., \& Sherman, D. K. (2007). Cultural differences in the impact of social support on psychological and biological stress responses. Psychological Science, 18(9), 831-837. doi:10.1111/j.1467-9280.2007.01987.x

Thoits, P. A. (1995). Stress, coping, and social support processes: Where are we? What next?. Journal of Health and Social Behavior, 53-79.

Tsai, W., Chiang, J. J., \& Lau, A. S. (2016). The effects of self-enhancement and selfimprovement on recovery from stress differ across cultural groups. Social Psychological and Personality Science, 7(1), 21-28. doi:10.1177/1948550615598380

Turner-Cobb, J. M., Gore-Felton, C., Marouf, F., Koopman, C., Kim, P., Israelski, D., \& Spiegel, D. (2002). Coping, social support, and attachment style as psychosocial correlates of adjustment in men and women with HIV/AIDS. Journal of Behavioral Medicine, 25(4), 337-353.

Turner-Cobb, J. M., Sephton, S. E., Koopman, C., Blake-Mortimer, J., \& Spiegel, D. (2000). Social support and salivary cortisol in women with metastatic breast cancer. Psychosomatic Medicine, 62(3), 337-345.

Uchino, B. N., Cacioppo, J. T., Malarkey, W., Glaser, R., \& Kiecolt-Glaser, J. K. (1995). Appraisal support predicts age-related differences in cardiovascular function in women. Health Psychology, 14(6), 556.

Uchino, B. N., \& Garvey, T. S. (1997). The availability of social support reduces cardiovascular reactivity to acute psychological stress. Journal of Behavioral Medicine, 20(1), 15-27. doi:10.1023/A:1025583012283

Wang, H. X., Mittleman, M. A., \& Orth-Gomer, K. (2005). Influence of social support on progression of coronary artery disease in women. Social Science \& Medicine, 60(3), 599-607. 
Wang, S., \& Lau, A. S. (2015). Mutual and non-mutual social support: Cultural differences in the psychological, behavioral, and biological effects of support seeking. Journal of Cross-Cultural Psychology, 46(7), 916-929. doi: $10.1177 / 0022022115592967$

Wang, S., Shih, J. H., Hu, A. W., Louie, J. Y., \& Lau, A. S. (2010). Cultural differences in daily support experiences. Cultural Diversity and Ethnic Minority Psychology, 16(3), 413-420. doi:10.1037/a0019885

Wells, K. (1980). Gender-role identity and psychological adjustment in adolescence. Journal of Youth and Adolescence, 9(1), 59-73. 


\section{Appendices}

\section{Appendix A: Screening Questionnaire}

Participant No.

The following questions ask about activities that you may or may not have done within the last hour. The rest of the questions are related to the study. Mark either the "Yes" or "No" box to the right of each question. If a question does not apply to you, mark "N/A". Please answer the following questions as best as you can.

1. Are you under the age of 18 ?

$\square$ Yes $\quad \square$ No

2. Did you smoke any cigarettes in the last hour?

$\square$ Yes $\quad \square$ No

3. Did you exercise in the last hour?

$\square$ Yes $\quad \square$ No

4. Did you eat any food in the last hour, including gum and mints? $\square$ Yes $\quad \square$ No

5. Did you drink anything in the last hour? (Except water)

$\square$ Yes $\quad \square$ No

6. Do you have any chronic inflammatory or neuroendocrine

$\square$ Yes $\quad \square$ No disorders?

7. Are you currently being treated for any Mood or Anxiety

$\square$ Yes $\quad \square$ No disorders or have been diagnosed with a Mood or Anxiety disorder within the past 3 months?

8. Are you pregnant?

$\square \mathrm{n} / \mathrm{a}$

$\square$ Yes

$\square$ No

9. Have you served in the military?

$\square$ Yes $\quad \square$ No 


\section{Appendix B: Debriefing Script}

This is the end of the study. I would like to thank you for your time and participation and tell you a little more about this study. This study examined the effects of differing cultural values on the effectiveness of social support as a coping mechanism against stress. We were also interested in the effects of gender roles during social support seeking and how both factors affect your response to stressful cognitive exercises. Although you might not have felt like it, you did great. During the speech, the judges are required not to respond or give any encouragement, so please do not feel like you did poorly. Furthermore, the video camera present in the interview room was only there for appearances, so don't worry, your speech was not recorded. I ask that you do not communicate the details of this study with other students, as they may need to participate in order to receive course credit or extra credit. This would also help us in collecting quality data to yield accurate results. We are also required to notify you about the counseling services available at SJSU. The SJSU counseling center is located in the Student Wellness Center on the third floor in room 300B. Again, I would like to thank you for your time and participation. Do you have any questions? (Answer any questions the participant may have). Please feel free to take a complimentary chocolate on your way out. Thank you again for your time and participation. 


\title{
Appendix C: Asian American Values Scale
}

\author{
Asian American Values Scale-Multidimensional (AAVS-M)
}

Items

\section{Collectivism}

The welfare of the group should be put before that of the individual.

One's efforts should be directed toward maintaining the well-being of the group first and the individual second.

One's personal needs should be second to the needs of the group.

The needs of the community should supersede those of the individual.

One need not always consider the needs of the group first. ${ }^{2}$

The group should be less important than the individual.

One need not sacrifice oneself for the benefit of the group. ${ }^{2}$

\section{Conformity to Norms}

One should recognize and adhere to the social expectations, norms, and practices.

One should adhere to the values, beliefs, and behaviors

that one's society considers normal and acceptable.

Conforming to norms provides one with identity.

One need not blend in with society.

Conforming to norms is the safest path to travel.

Conforming to norms provides order in the community.

One should not do something that is outside of the norm.

\section{Emotional Self-Control}

It is better to show emotions than to suffer quietly. ${ }^{2}$

One should be expressive with one's feelings.

Openly expressing one's emotions is a sign of strength. ${ }^{2}$

It is better to hold one's emotions inside than to burden others by expressing them.

It is more important to behave appropriately than to act on what one is feeling.

One should not express strong emotions.

One's emotional needs are less important than fulfilling one's responsibilities.

One should not act based on emotions. 
Asian American Values Scale-Multidimensional (AAVS-M)

Items

4. Family Recognition Through Achievement

One should achieve academically since it reflects on one's family.

Succeeding occupationally is an important way of making one's family proud.

Getting into a good school reflects well on one's family.

Failing academically brings shame to one's family.

One should go as far as one can academically and professionally on behalf of one's family.

One's academic and occupational reputation reflects the family's reputation.

Academic achievement should be highly valued among family members.

One's achievement and status reflect on the whole family.

Making achievements is an important way to show one's appreciation for one's family.

One's educational success is a sign of personal and familial character.

One should work hard so that one won't be a disappointment to one's family.

It is one's duty to bring praise through achievement to one's family.

Receiving awards for excellence need not reflect well on one's family. "

Children's achievements need not bring honor to their parents.

\section{Humility}

One should be able to brag about one's achievements.

One should be able to boast about one's achievement.

One should not sing one's own praises.

One should not openly talk about one's accomplishments.

One should be able to draw attention to one's accomplishments.

Being boastful should not be a sign of one's weakness and insecurity."

"Reverse-worded item. 


\section{Appendix D: Bem Sex Role Inventory}

Bem Sex Role Inventory

\begin{tabular}{|c|c|c|}
\hline Masculine Items & Feminine Items & Neutral items \\
\hline 49. Acts as a leader & 11. Affectionate & 51. Adaptable \\
\hline 46. Aggressive & 5. Cheerful & 36. Conceited \\
\hline 58. Ambitious & 50. Childlike & 9. Conscientious \\
\hline 22. Analytical & 32. Compassionate & 60. Conventional \\
\hline 13. Assertive & 53. Does not use harsh language & 45. Friendly \\
\hline 10. Athletic & 35. Eager to soothe hurt feelings & 15. Happy \\
\hline 55. Competitive & 20. Feminine & 3. Helpful \\
\hline 4. Defends own beliefs & 14. Flatterable & 48. Inefficient \\
\hline 37. Dominant & 59. Gentle & 24. Jealous \\
\hline 19. Forceful & 47. Gullible & 39. Likable \\
\hline 25. Has leadership abilities & 56. Loves children & 6. Moody \\
\hline 7. Independent & 17. Loyal & 21. Reliable \\
\hline 52. Individualistic & 26. Sensitive to the needs of others & 30. Secretive \\
\hline 31. Makes decisions easily & 8. Shy & 33. Sincere \\
\hline 40. Masculine & 38. Soft spoken & 42. Solemn \\
\hline 1. Self-reliant & 23. Sympathetic & 57. Tactful \\
\hline 34. Self-sufficient & 44. Tender & 12. Theatrical \\
\hline 16. Strong personality & 29. Understanding & 27. Truthful \\
\hline 43. Willing to take a stand & 41. Warm & 18. Unpredictable \\
\hline 28. Willing to take risks & 2. Yielding & 54. Unsystematic \\
\hline
\end{tabular}

Note. The number preceding each item reflects the position of each adjective as it actually appears on the Inventory. 


\section{Appendix E: Social Support Letter Writing Task}

Parent group: Think about one of your parents and write a letter to them, asking for advice on the speech you are about to deliver. As you write, think about how this person would respond to you. Remember, we will look over your letter only to make sure you have followed the task. You will have 3 minutes for this exercise.

Peer group: Think about one of your peers and write a letter to them, asking for advice on the speech you are about to deliver. As you write, think about how this person would respond to you. Remember, we will look over your letter only to make sure you have followed the task. You will have 3 minutes for this exercise.

No support group: Think and write about concerns you may have for the speech you are about to deliver. Remember, we will look over your letter only to make sure you have followed the task. You will have 3 minutes for this exercise. 